\title{
Roll- and pitch-plane coupled hydro-pneumatic suspension.
}

Part 1: Feasibility analysis and suspension properties

$$
\text { Dongpu Cao }{ }^{1 *} \text {, Subhash Rakheja }{ }^{2} \text { and Chun-Yi } \mathrm{Su}^{2}
$$

${ }^{1}$ Waterloo Center for Automotive Research (WatCAR), Department of Mechanical and Mechatronics Engineering, University of Waterloo, Waterloo, Ontario, Canada

${ }^{2}$ CONCAVE Research Center, Department of Mechanical and Industrial Engineering Concordia University, Montreal, Quebec, Canada

E_mails: dongpu@uwaterloo.ca; rakheja@alcor.concordia.ca; cysu@alcor.concordia.ca *Corresponding author. Fax: 1-514-848-8635 
Abstract: Passive fluidically-coupled suspensions have been considered to offer a promising alternative solution to the challenging design of vehicle suspension system. A theoretical foundation, however, has not been established for fluidically-coupled suspension to facilitate its broad applications to various vehicles. This first part of this study investigates the fundamental issues related to feasibility and properties of the passive full-vehicle interconnected hydro-pneumatic suspension configurations using both analytical and simulation techniques. Layouts of various interconnected suspension configurations are illustrated based on two novel hydro-pneumatic suspension strut designs, both of which provide a compact design with considerably large effective working area. A simplified measure, vehicle property index (VPI), is proposed to permit a preliminary evaluation of different interconnected suspension configurations using qualitative scaling of the bounce-, roll-, pitch- and warp-mode stiffness properties. Analytical formulations for the properties of unconnected and three selected X-coupled suspension configurations are derived, and simulation results are obtained to illustrate their relative stiffness and damping properties in the bounce-, roll-, pitch- and warp-mode. The superior design flexibility feature of the interconnected hydro-pneumatic suspension is also discussed through sensitivity analysis to a design parameter, namely the annular piston area of the strut. The results demonstrate that full-vehicle interconnected hydro-pneumatic suspension could provide enhanced roll- and pitch-mode stiffness and damping, while retaining the soft bounce- and warp-mode properties. Such interconnected suspension thus offers considerable potential in realizing enhanced decoupling among the different suspension modes. 
Keywords: vehicle dynamics; interconnected suspension; hydro-pneumatic coupling; feasibility analysis; suspension property; tuning flexibility

\section{Introduction}

Suspension design is known to strongly affect the forces and moments transmitted to the vehicle body and wheels, and thus the vehicle performance characteristics. The design of a road vehicle suspension, however, involves complex compromises among various measures related to ride vibration, handling and roll dynamics [1-4]. Analysis of a full-vehicle suspension system generally concerns four fundamental vibration modes, namely bounce, roll, pitch and warp [5-8]. The vertical ride comfort generally requires a soft bounce mode, while relatively stiff roll and pitch modes are beneficial for inhibiting vehicle attitude during steering and braking/traction maneuvers [9-11]. Moreover, it has also been well accepted that the suspension warp mode should be as soft as possible for improved roadholding performance [5-8]. These four fundamental modes of a vehicle with conventional passive suspension system, however, are strongly coupled.

Passive anti-roll bars help realize relatively higher roll stiffness and thus higher roll stability limits of road vehicles, particularly the commercial vehicles, without affecting the suspension bounce and pitch properties. The anti-roll bars, however, tend to add weight and increase the warp mode stiffness, which is undesirable in view of the vehicle roadholding performance $[5-8,37]$. Although active anti-roll bars could help realize a better compromise among different measures, their implementations have been limited due 
to the added cost and weight. A number of active suspension concepts and designs have also been proposed to achieve superior ride, handling and control performance, while their implantations are limited to applications justifying the associated additional power demand, complex packaging, weight and cost [e.g., 4, 12, 13].

Alternatively, a wide range of semi-active suspension designs have evolved over the past few decades, which have been shown to yield improved ride performance and stable behavior due to their energy dissipation properties, while requiring only minimal power [e.g., 2, 12, 13]. Such suspension concepts, however, are generally based on damping control alone and tend to deteriorate the ride vibration performance at higher frequencies, compared to the conventional passive suspensions $[12,13]$. Concepts in alternative passive suspension mechanisms that permit an improved compromise among the conflicting performance measures would be considered desirable. More specifically, a suspension design that permits independent tuning for the four fundamental modes (bounce, roll, pitch and warp) or partial decoupling of the modes in a positive manner could yield an enhanced design compromise.

A few studies on fluidically-coupled vehicle suspensions have shown that either within- or across-axle coupled wheel suspensions offer considerable potential to realize a full or partial decoupling among the fundamental suspension modes [14-18, 23-25]. The fluidic couplings could be conveniently realized in a suspension system involving hydro-pneumatic struts. Hydro-pneumatic struts, unlike the other suspension units, could offer compact design with integrated damping control, improved ride comfort, ride height 
leveling control, as well as semi-active/active control. The majority of the studies have analyzed the properties of such struts coupled in the roll plane [16-18, 23-25], while a few have also investigated the properties of struts coupled in the pitch plane [14, 15]. These studies have invariably shown that the roll- and pitch-plane coupled hydro-pneumatic wheel suspension struts could help realize considerably higher roll and pitch stiffness, respectively, with only a minimal change in the bounce stiffness. Furthermore, the hydraulic couplings yield far greater damping properties than the unconnected or pneumatically-coupled suspension struts. In addition, such couplings provide greater design flexibility in realizing desired properties through sizing of the strut chambers and the coupling [16]. The hydro-pneumatic suspension systems have been employed in heavy military vehicles for about half a century, and have been regarded to hold the most significant potential for commercial vehicle applications [4, 20-22]. Gunter et al. [21] and Gilmore [22] stated that the hydro-pneumatic suspension system has been selected as one of the key technologies in the development of future military vehicles.

A few studies have also demonstrated the potential performance benefits of some particular roll- and pitch-plane hydraulically-coupled suspension configurations, which however either employed semi-active control [19] or used additional passive mechanical springs in parallel with the coupled suspension $[5,26]$. The use of additional mechanical springs for load sharing would tend to reduce the benefit of interconnected suspension [25]. Multi-chamber hydro-pneumatic struts offer numerous possibilities for hydraulic or pneumatic interconnections between different chambers of the wheel struts in the roll as well as pitch planes. The resulting coupled suspension properties would be expected to 
depend on the interconnection configurations and paths, and type of fluid coupling (hydraulic or pneumatic). Some of these coupling configurations may be infeasible to ensure stability, and undesirable in view of the four fundamental modes.

In this study, two novel hydro-pneumatic strut design concepts are considered, which could yield various fluidic interconnections between different chambers of different wheel suspension struts in the roll as well as pitch planes. A number of full-vehicle coupled hydro-pneumatic suspension configurations involving four suspension-strut units are conceived. A simplified performance measure is proposed to evaluate feasibility of the proposed configurations. A generalized analytical model is formulated to analyze fundamental properties of a few selected feasible configurations, which are compared with an unconnected suspension configuration with and without anti-roll bars.

\section{Suspension Struts and Full-Vehicle Interconnections}

The schematic of a hydro-pneumatic strut design comprising only one gas chamber, referred to as 'single-gas-chamber strut $B$ ', is illustrated in Figure 1(a). The strut integrates a gas chamber and damping valves within the same unit to realize a compact design. The strut consists of a number of damping orifices in the main piston separating hydraulic fluid chamber 1 from chambers 2 and 3, while a floating piston isolates the hydraulic fluid in chamber 2 from the nitrogen gas in chamber 4 . The shim disc valves, consisting of shim packs, are employed in conjunction with constant area bleed orifices to achieve variable flow resistance and thus the damping force. Such compact strut design not only eliminates the external gas chamber and external damping valves, but offers a 
relatively larger effective working area and thus significantly lower operating pressure corresponding to a given load, compared to those reported in [5, 17-19, 23-26].

Figure 1(b) presents the schematic of a novel hydro-pneumatic strut design, referred to as 'strut $A$ ', which comprises two gas chambers. The chambers 3 and 4 of the strut contain nitrogen gas, while the damping orifices and valves within the main piston provide hydraulic flow resistance between chambers 1 and 2 . Similar to the strut $B$, the twin-gas-chamber strut also offers a compact design with a relatively large effective operating area [27, 28]. Under a compression stroke, the gas in chamber 4 undergoes compression and tends to dominate the vertical suspension stiffness property. The spring rate in rebound is mostly determined by the gas pressure in chamber 3 , which undergoes compression during rebound. The proposed twin-gas-chamber strut design thus offers considerable potential for realizing nearly symmetric spring rates in compression and rebound $[27,28]$.

Compared to the strut designs reported in [5, 17-19, 23-26], these two strut designs provide considerable flexibilities to conveniently realize various interconnections among different hydraulic/pneumatic chambers of the different struts [14].

\section{FIGURE 1}

\section{Feasibility Analysis of Full-Vehicle Interconnected Suspensions}

The proposed hydro-pneumatic struts could yield numerous full-vehicle coupled suspension configurations through interconnections among different chambers of the four struts. It has been shown that different interconnection configurations could yield 
considerably different stiffness and damping properties of the suspension in the bounce, roll and pitch modes [14-16]. Moreover, some of the configurations may not be feasible. As an example, Fig. 2 illustrates a possible full-vehicle interconnected suspension configuration involving eight interconnecting pipes among the four strut units in the rolland pitch-plane arrangement. It can be seen that this configuration yields only two independent pressures, and would not be feasible when load variations are involved. A methodology for assessing the feasibility of possible full-vehicle interconnected suspension configurations is thus explored on the basis of different measures that are described below.

\section{FIGURE 2}

\section{Equivalent Static Interconnected Suspension (ESIS):}

For a given or conceived interconnected struts configuration, an alternative interconnected suspension could be obtained by adding/removing one or several interconnecting pipes. If the resulting configuration yields the same static equilibrium under varying load conditions, the configuration is called an Equivalent Static Interconnected Suspension (ESIS) of the original configuration. A particular interconnected suspension configuration may have many possible ESIS configurations. Considerable differences, however, might be expected between the properties and dynamic responses of an ESIS and the original configuration.

\section{Least Equivalent Static Interconnected Suspension (LESIS):}

Of all the equivalent static interconnected configurations of an interconnected suspension, 
those with least number of interconnections are referred to as Least Equivalent Static Interconnected Suspension (LESIS) of the original suspension configuration. A given interconnected suspension configuration may have multiple LESIS configurations.

\section{Order of Least Equivalent Static Interconnected Suspension (OLESIS):}

The order of a LESIS is defined by the number of its interconnecting paths or pipes. For example, the configuration illustrated in Fig.2 possesses an order of 8, although it cannot be considered as a LESIS configuration.

\section{Degrees-of-Freedom (DOF) of an Interconnected Suspension:}

The minimum number of independent fluid pressures required to define the static equilibrium of an interconnected suspension is referred to its degrees of freedom (DOF). It can be seen that the configuration, illustrated in Fig. 2, possesses two-DOF. For a full-vehicle suspension configuration involving the proposed suspension struts (Fig. 1), the maximum DOF of an interconnected suspension could be eight. The sum of the order of its LESIS and the degrees of freedom would also be eight.

The load supported by the four suspension struts of an interconnected suspension in the roll- and pitch-plane arrangement could vary considerably, particularly in highway freight vehicles and many off-road vehicles. It has been shown that an interconnected suspension is most beneficial in enhancing its roll properties when the entire sprung load is fully supported by the interconnected struts [25]. It can thus be deduced that a minimum of four

independent fluid-pressures would be essential to obtain a static equilibrium of a full-vehicle interconnected suspension configuration, particularly when the load variations 
are expected to be large. This also defines the necessary condition of a feasible full-vehicle interconnected suspension configuration. A few examples of the feasible full-vehicle interconnected suspension configurations are described below.

Figure 3 presents two different feasible 4-DOF interconnected suspensions with four single-gas-chamber struts, shown in Fig. 1(a), involving hydraulic couplings. Figure 4 further illustrates two examples of feasible 4-DOF interconnected suspensions using four twin-gas-chamber struts, shown in Fig. 1(b), involving only pneumatic couplings. The examples of feasible 4-DOF interconnected suspensions using both types of struts are shown in Fig. 5, which involve hybrid fluidic couplings. A simple feasible configuration involving twin-gas struts and four pneumatic interconnecting paths is shown in Fig. 6. This configuration possesses 6-DOF. It can be seen that a number of feasible full-vehicle interconnected suspensions can be realized on the basis of the two hydro-pneumatic struts.

FIGURE 3

FIGURE 4

FIGURE 5

FIGURE 6

It would be impractical to investigate the properties and dynamic responses of all the possible configurations of full-vehicle interconnected suspensions in order to identify a more promising configuration. However, it was established that some of the full-vehicle interconnected configurations are simply a combination of the in-plane interconnected 
suspensions, whose properties have been reported in the earlier studies [14-16]. The reported properties of the roll- or pitch-plane interconnections could facilitate the preliminary selection and analysis of some of the full-vehicle interconnected configurations.

\subsection{Identification of Feasible Interconnected Suspension Configurations}

A total of 22 different full-vehicle suspension configurations, including four unconnected (U1 U4) and 18 interconnected $(\mathrm{C} 1 \sim \mathrm{C} 18)$, are selected in this study for the preliminary analyses in view of their properties. Figure 7 illustrates the four unconnected suspension configurations, where configurations $\mathrm{U} 1$ and $\mathrm{U} 2$ comprise four single- and twin-gas-chamber struts, respectively. The configurations U3 and U4 comprise two singleand two twin-gas-chamber struts, as shown in Fig. 7.

\section{FIGURE 7}

Figure 8 presents the four 4-DOF full-vehicle interconnected suspension configurations that involve diagonal fluidic couplings (or X-couplings) among the different strut units. Configurations $\mathrm{C} 1$ and $\mathrm{C} 2$ employ single- and twin-gas chamber struts, respectively, with diagonal hydraulic and pneumatic interconnections. Configurations C3 and C4 employ combinations of the two types of struts with hybrid fluidic interconnections. Figure 9 presents four 4-DOF full-vehicle interconnected configurations (C5 to C8) that involve roll-plane fluidic couplings, where configurations C5 and C6 employ four single-and twin-gas-chamber struts, respectively, with hydraulic and pneumatic interconnections in the roll-plane alone. Configurations $\mathrm{C} 7$ and $\mathrm{C} 8$ are conceived through either hydraulic or pneumatic couplings of the single- or dual-gas-chamber struts in the roll plane. 


\section{FIGURE 8}

\section{FIGURE 9}

Four different configurations involving pneumatic couplings in the pitch-plane alone are shown in Fig. 10. Configurations $\mathrm{C} 9$ and $\mathrm{C} 10$ are realized by two pitch-plane pneumatic interconnections between the chambers 4 and 3 of front- and rear-struts, respectively. The configuration C11 employs two twin- and single-gas-chamber struts in the front and rear, respectively, with pitch-plane pneumatic interconnections between the chambers 4 of the front- and rear-struts. Configuration $\mathrm{C} 12$ includes two single- and two twin-gas-chamber struts in the front and rear with pitch-plane pneumatic interconnections between chambers 4 of the front- and rear-struts. Two alternate configurations (C13 and C14) involving pneumatic X-couplings of the twin-gas chamber struts are shown in Fig. 11, which possess 6-DOF. Figure 12 illustrates another two 4-DOF configurations, C15 and C16, employing single- and twin-gas chamber struts, respectively, with relatively more complex couplings in the roll as well as pitch planes. Figure 13 further illustrates two 4-DOF interconnected suspension configurations, $\mathrm{C} 17$ and $\mathrm{C} 18$, which involve pitch-plane hydraulic and pneumatic couplings, respectively. Configuration C17 involves hydraulic interconnections between the front and rear single-gas-chamber struts, while C18 employs couplings between the front and rear twin-gas-chamber struts.

FIGURE 10

FIGURE 11 


\section{FIGURE 12}

\section{FIGURE 13}

The design and tuning of a full-vehicle suspension system generally concerns four fundamental modes: bounce, roll, pitch and warp [5-8]. A simplified composite measure of the four mode stiffness properties, referred to as vehicle property index (VPI), is proposed to facilitate the relative analyses of the above selected full-vehicle suspension configurations, such that:

$$
V P I=\alpha_{v}\left|K_{v}-K_{v o}\right|+\alpha_{R}\left|K_{R}-K_{R o}\right|+\alpha_{P}\left|K_{P}-K_{P o}\right|+\alpha_{\psi}\left|K_{\psi}-K_{\psi o}\right|
$$

where $K_{v o}, K_{R o}, K_{P o}$ and $K_{\psi o}$ represent the desirable suspension bounce-, roll-, pitch- and warp-mode stiffness properties for a road vehicle, respectively. $K_{v}, K_{R}, K_{P}$ and $K_{\psi}$ represent actual suspension bounce-, roll-, pitch- and warp-mode stiffness properties of a given interconnected configuration, respectively. $\alpha_{v}, \alpha_{R}, \alpha_{P}$ and $\alpha_{\psi}$ are corresponding weighting coefficients for the four modes stiffness constants, respectively. Considering the complexity of defining the desired values, a qualitative scale ranging from 1 to 5 is assigned to each stiffness constant in an attempt to obtain a preliminary analysis of relative properties of the configurations. The scale 5 refers to the highest stiffness, while the scale 1 refers to the lowest stiffness of a mode. For a typical heavy road vehicle, greater emphasis is placed on the roll stiffness compared to the bounce stiffness, while relatively lower emphasis is given to the pitch and warp stiffness by selecting weighting constants as: $\alpha_{r}=0.5, \alpha_{b}=0.3$, and $\alpha_{p}=\alpha_{w}=0.1$. For heavy vehicles, relatively higher values of roll and

pitch stiffness are considered desirable [1, 9-11], while a medium bounce stiffness would be appropriate. Moreover, a lower value of warp stiffness would also be adequate [5-8]. 
The stiffness constants $K_{v o}, K_{R o}, K_{P o}$ and $K_{\psi o}$ are thus assigned 3, 5, 5 and 1, respectively, on the qualitative scale for preliminary selection of the desirable suspension configurations. On the basis of the chosen rule, a configuration with a lower VPI would be considered favorable, which could yield relatively soft vertical ride, enhanced roadholding quality, as well as improved anti-roll and anti-pitch properties.

Table 1 summarizes the vehicle property indices (VPIs) of the selected full-vehicle suspension configurations, either interconnected or unconnected, on the basis of the above-stated rule. The table also lists the VPIs of unconnected configurations coupled with anti-roll bars, referred to as U1bar to U4bar. The results clearly show that different full-vehicle interconnected suspension systems could yield considerably different suspension stiffness properties. Moreover, the configurations $\mathrm{C} 1$ to $\mathrm{C} 4$ (Fig. 8) employing fluidic X-couplings yield the lowest VPIs among the considered configurations, suggesting that the X-couplings would most likely to offer considerable potential benefits for a typical heavy vehicle. The properties of these configurations are thus evaluated through formulations of strut forces in term of suspension stiffness and damping properties. The properties are also compared with those of the unconnected configuration U1 and U1bar. In the following sections, the interconnected suspension configurations $\mathrm{C} 1 \sim \mathrm{C} 3$, while hybrid interconnected suspension configuration $\mathrm{C} 4$ would be expected to yield similar performance to configuration $\mathrm{C} 3$. The analyses presented in the following sections are also used to demonstrate the effectiveness of the proposed simplified VPI measure for preliminary evaluations of the full-vehicle interconnected suspension design.

\section{TABLE 1}




\section{Formulations of Strut Forces}

Although the three coupled suspension configurations $\mathrm{C} 1 \sim \mathrm{C} 3$ involve complex cross-axle fluid flows across different suspension struts, the forces developed by each strut can be conveniently derived using a generalized model presented in [14]. The generalized model permits the evaluation of restoring and dissipative suspension forces due to hydro-pneumatic struts, uncoupled or coupled in the roll and/or pitch planes. The primary assumptions in the modeling include: (i) turbulent flows through damping orifices between the hydraulic chambers (1 and 2) within the same strut; (ii) laminar flows through hydraulic interconnections between the connected struts; (iii) polytropic gas process; (iv) incompressible hydraulic fluid; (v) negligible leakage flows; (vi) negligible thermal expansion of the struts and hydraulic fluid; (vii) negligible friction between the pistons seals and the cylinder; and (viii) negligible floating piston dynamics. It has been shown that the bulk modulus of the hydraulic fluid is quite large, and the consideration of fluid compressibility yields only negligible influence on the stiffness properties [16, 29].

The mathematical formulations for the strut forces of configurations $\mathrm{C} 1$ and $\mathrm{U} 1$ alone are presented as examples, while those of the other configurations could be developed in a similar manner using the generalized model described in [29]. The configuration C1 involves couplings between the hydraulic fluid chambers 1 and 3 of the four suspension struts, as shown in Figure 8. Chambers 1 and 3 of the rear-left strut are coupled to 3 and 1 of the front-right strut, respectively, and these couplings are referred to as $1 r l 3 f r$ and $3 r l l f r$, 
respectively. In a similar manner, the chambers 1 and 3 of the rear-right strut are coupled to 3 and 1 of the front-left strut, respectively, and referred to as $1 \mathrm{rr} 3 \mathrm{fl}$ and $3 \mathrm{rr} / \mathrm{fl}$, respectively. The forces developed by the front-left $(f l)$ and -right $(f r)$ struts in the interconnected suspension C1 are derived using the methodology described in [29], and expressed as:

$$
\begin{aligned}
& F_{f l}=P_{0}\left[A_{1 f f}\left(\frac{V_{40 f l}}{V_{40 f l}+A_{1 f l} x_{f l}-A_{3 r r} x_{r r}}\right)^{n}-A_{3 f l}\left(\frac{V_{40 r r}}{V_{40 r r}+A_{1 r r} x_{r r}-A_{3 f l} x_{f l}}\right)^{n}\right] \\
& -P_{0} A_{2 f l}-\frac{\rho A_{1 f l}\left|A_{1 f f} \dot{x}_{f f l}-A_{3 r r} \dot{x}_{r r}\right|\left(A_{1 f f} \dot{x}_{f l}-A_{3 r r} \dot{x}_{r r r}\right)}{2 C_{d}^{2} a_{12 f l}^{2}}-\frac{128 \mu L A_{3 f l}^{2} \dot{x}_{f l}}{\pi D^{4}}+\frac{\rho A_{3 f l}\left|A_{1 r r} \dot{x}_{r r}-A_{3 f l} \dot{x}_{f l}\right|\left(A_{1 r r} \dot{x}_{r r}-A_{3 f l} \dot{x}_{f f l}\right)}{2 C_{d}^{2} a_{12 r r}^{2}} \\
& F_{f r r}=P_{0}\left[A_{1 f r}\left(\frac{V_{40 f r}}{V_{40 f r}+A_{1 f r} x_{f r}-A_{3 r l} x_{r l}}\right)^{n}-A_{3 f r}\left(\frac{V_{40 r l}}{V_{40 r l}+A_{1 r l} x_{r l}-A_{3 f r} x_{f r}}\right)^{n}\right] \\
& -P_{0} A_{2 f r}-\frac{\rho A_{1 f r}\left|A_{1 f r} \dot{x}_{f r}-A_{3 r l} \dot{x}_{r l}\right|\left(A_{1 f r} \dot{x}_{f r}-A_{3 r l} \dot{x}_{r l}\right)}{2 C_{d}^{2} a_{12 f r}^{2}}-\frac{128 \mu L A_{3 f r}^{2} \dot{x}_{f r}}{\pi D^{4}}+\frac{\rho A_{3 f r}\left|A_{1 r l} \dot{x}_{r l}-A_{3 f r} \dot{x}_{f r}\right|\left(A_{1 r l} \dot{x}_{r l}-A_{3 f r} \dot{x}_{f r}\right)}{2 C_{d}^{2} a_{12 r l}^{2}}
\end{aligned}
$$

In a similar manner, the forces developed by the rear struts are also derived, such that:

$$
\begin{aligned}
& F_{r l}=P_{0}\left[A_{1 r l}\left(\frac{V_{40 r l}}{V_{40 r l}+A_{1 r l} x_{r l}-A_{3 f r} x_{f r}}\right)^{n}-A_{3 r l}\left(\frac{V_{40 f r}}{V_{40 f r}+A_{1 f r} x_{f r}-A_{3 r l} x_{r l}}\right)^{n}\right] \\
& -P_{0} A_{2 r l}-\frac{\rho A_{1 r l} \mid A_{1 r l} \dot{x}_{r l}-A_{3 f r} \dot{x}_{f r}\left(A_{1 r l} \dot{x}_{r l}-A_{3 f r} \dot{x}_{f r}\right)}{2 C_{d}^{2} a_{12 r l}^{2}}-\frac{128 \mu L A_{3 r l}^{2} \dot{x}_{r l}}{\pi D^{4}}+\frac{\rho A_{3 r l}\left|A_{1 f r} \dot{x}_{f r}-A_{3 r l} \dot{x}_{r l}\right|\left(A_{1 f r} \dot{x}_{f r}-A_{3 r l} \dot{x}_{r l}\right)}{2 C_{d}^{2} a_{12 f r}^{2}} \\
& F_{r r}=P_{0}\left[A_{1 r r}\left(\frac{V_{40 r r}}{V_{40 r r}+A_{1 r r} x_{r r}-A_{3 f l} x_{f l}}\right)^{n}-A_{3 r r}\left(\frac{V_{40 f l}}{V_{40 f l}+A_{1 f l} x_{f l}-A_{3 r r} x_{r r}}\right)^{n}\right] \\
& -P_{0} A_{2 r r}-\frac{\rho A_{1 r r}\left|A_{1 r r} \dot{x}_{r r}-A_{3 f l} \dot{x}_{f l}\right|\left(A_{1 r r} \dot{x}_{r r}-A_{3 f l} \dot{x}_{f l}\right)}{2 C_{d}^{2} a_{12 r r}^{2}}-\frac{128 \mu L A_{3 r r}^{2} \dot{x}_{r r r}}{\pi D^{4}}+\frac{\rho A_{3 r r}\left|A_{1 f l} \dot{x}_{f l}-A_{3 r r} \dot{x}_{r r}\right|\left(A_{1 f l} \dot{x}_{f l}-A_{3 r r} \dot{x}_{r r}\right)}{2 C_{d}^{2} a_{12 f l}^{2}}
\end{aligned}
$$

In the above formulations, $F_{i}$ is the dynamic force developed by strut $i(i=f l, f r, r l, r r), A_{j i}$ is 
the effective piston area reflected on the chamber $j(j=1,2,3)$ side of the strut $i, P_{0}$ is the static charge pressure, $n$ is the polytropic constant, $x_{i}$ and $\dot{x}_{i}$ are the relative displacement and velocity across strut $i$, respectively, assuming small motions with positive direction being upward. $V_{40 i}$ is the initial volume of gas in chamber 4 of strut $i, C_{d}$ is the discharge coefficient, $\rho$ is mass density of the hydraulic fluid, $\mu$ represents the dynamic viscosity of the hydraulic fluid, and $L$ and $D$ represent the length and diameter of the interconnecting pipes, respectively. $a_{12 i}$ is the effective orifice area due to bleed orifices and damping valves of strut $i$. Equation (2) clearly shows that hydraulic interconnections in the $\mathrm{C} 1$ configuration exhibit strong couplings in the stiffness as well as damping terms.

Variations in damping forces could be realized by varying the effective flow areas of damping valves. Modeling of damping variations in a piecewise-linear manner [30] would yield non-differentiable variations in the fluid pressures in the vicinity of the transition pressures, a refined model has thus been used to smoothen the flow variations around the transition regions [16]. Asymmetric damping force in compression/rebound motions could be achieved by selecting different flow areas, threshold values of pressures in compression and extension $\left(P_{c s l}, P_{e s l}\right)$, and the peak pressure differentials in compression and extension $\left(P_{c s h}, P_{e s h}\right)$, such that:

$$
a_{12 i}=\left\{\begin{array}{cc}
a_{12 c} & -P_{e s l} \leq P_{12 i} \leq P_{c s l} \\
a_{12 c}+a_{12 v c}\left\{\frac{\left(\left|P_{12 i}\right|-P_{c s l}\right)}{P_{c s h}-P_{c s l}}-\frac{1}{2 \pi} \sin \left[\frac{2 \pi\left(\left|P_{12 i}\right|-P_{c s l}\right)}{P_{c s h}-P_{c s l}}\right]\right\} & P_{c s l}<P_{12 i} \leq P_{c s h} \\
a_{12 c}+a_{12 v c} & P_{12 i}>P_{c s h} \\
a_{12 c}+a_{12 v e}\left\{\frac{\left(\left|P_{12 i}\right|-P_{e s l}\right)}{P_{e s h}-P_{e s l}}-\frac{1}{2 \pi} \sin \left[\frac{2 \pi\left(\left|P_{12 i}\right|-P_{e s l}\right)}{P_{e s h}-P_{e s l}}\right]\right\} & P_{e s l}<P_{12 i} \leq P_{e s h} \\
a_{12 c}+a_{12 v e} & P_{12 i}>P_{e s h}
\end{array}\right.
$$


where $a_{12 c}$ is the bleed orifice area, $a_{12 v c}$ and $a_{12 v e}$ are the maximum effective flow areas of valves in compression and rebound, respectively. $P_{c s l}$ and $P_{e s l}$ are the lower limits of pressure differentials or threshold values in compression and rebound, which cause deflections of shim discs to initiate valve opening, and $P_{c s h}$ and $P_{e s h}$ are the pressures that cause maximum valve openings, namely $a_{12 v c}$ and $a_{12 v e}$. The symmetric damping property can also be easily achieved by letting: $P_{c s l}=P_{e s l}, P_{c s h}=P_{e s h}$ and $a_{12 v c}=a_{12 v e}$.

The dynamic strut forces due to the unconnected strut suspension configuration U1 are developed in a similar manner, such that [29]:

$$
F_{i}=P_{0} A_{2 i}\left[\left(\frac{V_{40 i}}{V_{40 i}+A_{2 i} x_{i}}\right)^{n}-1\right]-\frac{\rho A_{2 i}^{3}\left|\dot{x}_{i}\right| \dot{x}_{i}}{2 C_{d}^{2} a_{12 i}^{2}}-\frac{\rho A_{3 i}^{3}\left|\dot{x}_{i}\right| \dot{x}_{i}}{2 C_{d}^{2} a_{13 i}^{2}} \quad(i=f l, f r, r l, r r)
$$

\section{Formulations of Suspension Properties}

The fundamental property of a vehicle suspension system can be effectively evaluated using two approaches [14-16, 18, 31, 32]. An analytical approach based upon coupled vibration-modes of a vehicle, e.g. coupled roll and bounce modes, in an ideal manner that neglects the influence of chassis structural-deflection modes and suspension kinematics, has been used to determine the stiffness and damping characteristics of the suspension systems [14-16, 18]. Considering the strong coupling effects of the vibration-modes of a vehicle with a suspension, the approach may be considered theoretically accurate [33]. This approach, however, is quite sensitive to variations in the vehicle inertial and geometry parameters, and may not be efficient for evaluating the full-vehicle suspension system involving complex couplings among the bounce-, roll-, pitch- and warp-modes. An 
alternative approach assumes a fixed vehicle body (or sprung mass) to appropriately reduce the contributions of couplings among various vibrations-modes of the vehicle body and therefore facilitate the characterization of stiffness properties of suspension system in individual modes $[31,32]$. This method can be considered to be more suspension-oriented, and less sensitive to variations in the vehicle inertial parameters. Moreover, this approach is far simpler than the former method due to the absence or reduction in coupling between the various vehicle vibration-modes. The properties of the interconnected and unconnected configurations are thus evaluated using the latter approach using the simplified model of the struts with fixed sprung mass, as shown in Fig. 14. In this model, the four struts are subject to four independent excitations $\left(X_{f l}, \quad X_{f r}, \quad X_{r l}, X_{r r}\right)$ representing the compression/extension of the struts.

\section{FIGURE 14}

\subsection{Stiffness Properties}

\section{BOUNCE MODE:}

The bounce-mode stiffness properties (or suspension rate) of the unconnected and interconnected suspensions are evaluated by letting $X_{f l}=X_{f r}=X_{r l}=X_{r r}=x$. The suspension rates, $k_{v f}$ and $k_{v r}$, of the front and rear suspensions, respectively, are derived from:

$$
k_{v f}=-\frac{\partial\left(F_{f l}+F_{f r}\right)}{d x} ; k_{v r}=-\frac{\partial\left(F_{r l}+F_{r r}\right)}{d x}
$$

The bounce-mode stiffness of the total suspension system is simply the sum of the suspension rates of its front and rear suspensions. 


\section{ROLL MODE:}

The roll-mode stiffness property of a full-vehicle suspension, whether unconnected or interconnected, is evaluated by letting $X_{f l}=-X_{f r}=X_{r l}=-X_{r r}=x$. The roll-mode stiffness properties, $k_{R f}$ and $k_{R r}$, of the front and rear suspensions, respectively, are evaluated from:

$$
k_{R f}=-\frac{\partial M_{f}}{d \theta_{f}}=-\frac{\partial\left(F_{f l}-F_{f r}\right)}{d x} l_{s f}^{2} ; \quad k_{R r}=-\frac{\partial M_{r}}{d \theta_{r}}=-\frac{\partial\left(F_{r l}-F_{r r}\right)}{d x} l_{s r}^{2}
$$

where $M_{f}$ and $M_{r}$ are the roll moments due to forces developed in the front- and rear suspension struts, respectively, $\theta_{f}$ and $\theta_{r}$ are respective roll motions, and $l_{s f}$ and $l_{s r}$ are the half suspension spacing. The roll stiffness of a full-vehicle suspension system is the sum of the roll stiffnesses due to the front and rear suspensions.

\section{PITCH MODE:}

The pitch-mode stiffness property $\left(k_{P}\right)$ of a full-vehicle suspension, either unconnected or interconnected, is evaluated by letting $X_{f l}=X_{f r}=-X_{r l}=-X_{r r}=x$, such that:

$$
\left.k_{p}=-\frac{\partial M_{p}}{d \varphi}=-\frac{\partial\left(\left(F_{f l}+F_{f)}\right) l-{ }_{f}\left(F+F_{l}\right) l\right.}{d x}\right), \frac{\left(l_{f}+l_{r}\right)}{2}
$$

where $M_{p}$ is the pitch moments due to forces developed in the front- and rear suspension struts, $\varphi$ is the vehicle pitch motion, and $l_{f}$ and $l_{r}$ are longitudinal distances of the front and rear suspensions, respectively, with respect to the sprung mass center of gravity (c.g.). 


\section{WARP MODE:}

The warp-mode stiffness property $\left(k_{w}\right)$ of a full-vehicle suspension is assessed by letting $X_{f l}=-X_{f r}=-X_{r l}=X_{r r}=x$. The warp-mode stiffness can be simply expressed as the front and rear suspension roll stiffness in series, such that [37]:

$$
k_{\psi f}=-\frac{\partial M_{f}}{d \psi_{f}}=-\frac{\partial\left(F_{f l}-F_{f r}\right)}{d x} l_{s f}^{2} ; \quad k_{\psi r}=-\frac{\partial M_{r}}{d \psi_{r}}=-\frac{\partial\left(F_{r l}-F_{r r}\right)}{d x} l_{s r}^{2} ; \quad k_{w}=\frac{k_{\psi f} k_{\psi r}}{k_{\psi f}+k_{\psi r}}
$$

where $k_{\psi f}$ and $k_{\psi r}$ are warp-mode stiffness constants of the front and real suspension struts, respectively, and $\psi_{f}$ and $\psi_{r}$ are respective warp motions.

\subsection{Interconnected Suspension (C1)}

The bounce-mode stiffness properties of the X-coupled suspension configuration $\mathrm{C} 1$ are derived using Eqs. (2) and (5), such that:

$$
\begin{aligned}
& k_{v f}=2 n P_{0} V_{40 f}^{n} \frac{A_{1 f}^{2}-A_{1 f} A_{3 r}}{\left[V_{40 f}+A_{1 f} x-A_{3 r} x\right]^{n+1}}+2 n P_{0} V_{40 r}^{n} \frac{A_{3 f}^{2}-A_{1 r} A_{3 f}}{\left[V_{40 r}+A_{1 r} x-A_{3 f} x\right]^{n+1}} \\
& k_{v r}=2 n P_{0} V_{40 r}^{n} \frac{A_{1 r}^{2}-A_{1 r} A_{3 f}}{\left[V_{40 r}+A_{1 r} x-A_{3 f} x\right]^{n+1}}+2 n P_{0} V_{40 f}^{n} \frac{A_{3 r}^{2}-A_{1 f} A_{3 r}}{\left[V_{40 f}+A_{1 f} x-A_{3 r} x\right]^{n+1}}
\end{aligned}
$$

The roll-mode stiffness properties of the suspension configuration $\mathrm{C} 1$ are derived from Eqs. (2) and (6), such that: 


$$
\begin{aligned}
& k_{R f}=n P_{0} V_{40 f}^{n} l_{s f}^{2} \frac{A_{1 f}^{2}+A_{1 f} A_{3 r}}{\left[V_{40 f}+A_{1 f} x+A_{3 r} x\right]^{n+1}}+n P_{0} V_{40 r}^{n} l_{s f}^{2} \frac{A_{3 f}^{2}+A_{1 r} A_{3 f}}{\left[V_{40 r}-A_{1 r} x-A_{3 f} x\right]^{n+1}} \\
& +n P_{0} V_{40 f}^{n} l_{s f}^{2} \frac{A_{1 f}^{2}+A_{1 f} A_{3 r}}{\left[V_{40 f}-A_{1 f} x-A_{3 r} x\right]^{n+1}}+n P_{0} V_{40 r}^{n} l_{s f}^{2} \frac{A_{3 f}^{2}+A_{1 r} A_{3 f}}{\left[V_{40 r}+A_{1 r} x+A_{3 f} x\right]^{n+1}} \\
& k_{R r}=n P_{0} V_{40 r}^{n} l_{s r}^{2} \frac{A_{1 r}^{2}+A_{1 r} A_{3 f}}{\left[V_{40 r}+A_{1 r} x+A_{3 f} x\right]^{n+1}}+n P_{0} V_{40 f}^{n} l_{s r}^{2} \frac{A_{3 r}^{2}+A_{1 f} A_{3 r}}{\left[V_{40 f}-A_{1 f} x-A_{3 r} x\right]^{n+1}} \\
& +n P_{0} V_{40 r}^{n} l_{s r}^{2} \frac{A_{1 r}^{2}+A_{1 r} A_{3 f}}{\left[V_{40 r}-A_{1 r} x-A_{3 f} x\right]^{n+1}}+n P_{0} V_{40 f}^{n} l_{s r}^{2} \frac{A_{3 r}^{2}+A_{1 f} A_{3 r}}{\left[V_{40 f}+A_{1 f} x+A_{3 r} x\right]^{n+1}}
\end{aligned}
$$

The pitch-mode stiffness property of configuration C1 is derived from Eqs (2) and (7), such that:

$$
\begin{aligned}
& k_{P}=n P_{0} l_{f}\left(l_{f}+l_{r}\right)\left\{V_{40 f}^{n} \frac{A_{1 f}^{2}+A_{1 f} A_{3 r}}{\left[V_{40 f}+A_{1 f} x+A_{3 r} x\right]^{n+1}}+V_{40 r}^{n} \frac{A_{3 f}^{2}+A_{1 r} A_{3 f}}{\left[V_{40 r}-A_{1 r} x-A_{3 f} x\right]^{n+1}}\right\} \\
& +n P_{0} l_{r}\left(l_{f}+l_{r}\right)\left\{V_{40 r}^{n} \frac{A_{1 r}^{2}+A_{1 r} A_{3 f}}{\left[V_{40 r}-A_{1 r} x-A_{3 f} x\right]^{n+1}}+V_{40 f}^{n} \frac{A_{3 r}^{2}+A_{1 f} A_{3 r}}{\left[V_{40 f}+A_{1 f} x+A_{3 r} x\right]^{n+1}}\right\}
\end{aligned}
$$

The formulations of the warp-mode stiffness properties of the suspension $\mathrm{C} 1$ are derived in a similar manner from Equations (2) and (8), such that:

$$
\begin{aligned}
& k_{\psi f}=n P_{0} V_{40 f}^{n} l_{s f}^{2} \frac{A_{1 f}^{2}-A_{1 f} A_{3 r}}{\left[V_{40 f}+A_{1 f} x-A_{3 r} x\right]^{n+1}}+n P_{0} V_{40 r}^{n} r_{s f}^{2} \frac{A_{3 f}^{2}-A_{1 r} A_{3 f}}{\left[V_{40 r}+A_{1 r} x-A_{3 f} x\right]^{n+1}} \\
& +n P_{0} V_{40 f}^{n} l_{s f}^{2} \frac{A_{1 f}^{2}-A_{1 f} A_{3 r}}{\left[V_{40 f}-A_{1 f} x+A_{3 r} x\right]^{n+1}}+n P_{0} V_{40 r}^{n} l_{s f}^{2} \frac{A_{3 f}^{2}-A_{1 r} A_{3 f}}{\left[V_{40 r}-A_{1 r} x+A_{3 f} x\right]^{n+1}} \\
& k_{\psi r}=n P_{0} V_{40 r}^{n} l_{s r}^{2} \frac{A_{1 r}^{2}-A_{1 r} A_{3 f}}{\left[V_{40 r}+A_{1 r} x-A_{3 f} x\right]^{n+1}}+n P_{0} V_{40 f}^{n} l_{s r}^{2} \frac{A_{3 r}^{2}-A_{1 f} A_{3 r}}{\left[V_{40 f}+A_{1 f} x-A_{3 r} x\right]^{n+1}} \\
& +n P_{0} V_{40 r}^{n} l_{s r}^{2} \frac{A_{1 r}^{2}-A_{1 r} A_{3 f}}{\left[V_{40 r}-A_{1 r} x+A_{3 f} x\right]^{n+1}}+n P_{0} V_{40 f}^{n} l_{s r}^{2} \frac{A_{3 r}^{2}-A_{1 f} A_{3 r}}{\left[V_{40 f}-A_{1 f} x+A_{3 r} x\right]^{n+1}}
\end{aligned}
$$


and

$$
k_{w}=\frac{k_{\psi f} k_{\psi r}}{k_{\psi f}+k_{\psi r}}
$$

\subsection{Unconnected Suspension (U1)}

The vertical- $\left(k_{v i}\right)$, roll- $\left(k_{R i}\right)$, pitch- $\left(k_{P}\right)$, and warp- $\left(k_{w}\right)$ mode stiffness properties of the unconnected configuration U1 are derived in a similar manner and expressed as:

$$
\begin{aligned}
& k_{v i}=2 n P_{0} V_{40 i}^{n} \frac{A_{2 i}^{2}}{\left[V_{40 i}+A_{2 i} x\right]^{n+1}} \quad(i=f, r) \\
& k_{R i}=n P_{0} V_{40 i}^{n} l_{s i}^{2} \frac{A_{2 i}^{2}}{\left[V_{40 i}+A_{2 i} x\right]^{n+1}}+n P_{0} V_{40 i}^{n} l_{s i}^{2} \frac{A_{2 i}^{2}}{\left[V_{40 i}-A_{2 i} x\right]^{n+1}} \quad(i=f, r) \\
& k_{P}=n P_{0} V_{40 f}^{n} l_{f}\left(l_{f}+l_{r}\right) \frac{A_{2 f}^{2}}{\left[V_{40 f}+A_{2 f} x\right]^{n+1}}+n P_{0} V_{40 r}^{n} l_{r}\left(l_{f}+l_{r}\right) \frac{A_{2 r}^{2}}{\left[V_{40 r}-A_{2 r} x\right]^{n+1}} \\
& k_{\psi i}=n P_{0} V_{40 i}^{n} l_{s i}^{2} \frac{A_{2 i}^{2}}{\left[V_{40 i}+A_{2 i} x\right]^{n+1}}+n P_{0} V_{40 i}^{n} l_{s i}^{2} \frac{A_{2 i}^{2}}{\left[V_{40 i}-A_{2 i} x\right]^{n+1}} \quad(i=f, r) ; \text { and } \\
& k_{w}=\frac{k_{\psi f} k_{\psi r}}{k_{\psi f}+k_{\psi r}}
\end{aligned}
$$

The analytical formulations for the stiffness properties of other suspension configurations can also be conveniently developed in a similar manner [29].

\subsection{Damping Properties}


The damping properties of the full-vehicle suspension configurations are also evaluated similar to the stiffness properties, where the displacement inputs are replaced by the velocity inputs. The four vibration-mode damping properties of the selected full-vehicle suspension configurations, however, are expressed by the dissipative components of the strut forces in each mode, using Eqs. (2) and (4). The benefit of employing damping valves in realizing variable damping properties could be emphasized by selecting relatively large-diameter connecting pipes. The damping due to interconnecting pipes would therefore be much smaller compared to that due to flows through chambers 1 and 2 , as observed from Eq. (2).

The vertical damping forces developed by the front struts in the X-coupled configuration $\mathrm{C} 1$ are directly deduced from Eq. (2), by considering identical velocities of all the struts, such that:

$$
\begin{aligned}
F_{d v f l} & \approx \frac{\rho A_{1 f l}\left|A_{1 f l} \dot{x}-A_{3 r r} \dot{x}\right|\left(A_{1 f l} \dot{x}-A_{3 r r} \dot{x}\right)}{2 C_{d}^{2} a_{12 f l}^{2}}-\frac{\rho A_{3 f l}\left|A_{1 r r} \dot{x}-A_{3 f l} \dot{x}\right|\left(A_{1 r r} \dot{x}-A_{3 f l} \dot{x}\right)}{2 C_{d}^{2} a_{12 r r}^{2}} \\
F_{d v f r} & \approx \frac{\rho A_{1 f r}\left|A_{1 f r} \dot{x}-A_{3 r l} \dot{x}\right|\left(A_{1 f r} \dot{x}-A_{3 r l} \dot{x}\right)}{2 C_{d}^{2} a_{12 f r}^{2}}-\frac{\rho A_{3 f r}\left|A_{1 r l} \dot{x}-A_{3 f r} \dot{x}\right|\left(A_{1 r l} \dot{x}-A_{3 f r} \dot{x}\right)}{2 C_{d}^{2} a_{12 r l}^{2}}
\end{aligned}
$$

In a similar manner, the vertical damping forces due to rear suspension struts can be expressed as:

$$
\begin{gathered}
F_{d v r l} \approx \frac{\rho A_{1 r l}\left|A_{1 r l} \dot{x}-A_{3 f r} \dot{x}\right|\left(A_{1 r l} \dot{x}-A_{3 f r} \dot{x}\right)}{2 C_{d}^{2} a_{12 r l}^{2}}-\frac{\rho A_{3 r l}\left|A_{1 f r} \dot{x}-A_{3 r l} \dot{x}\right|\left(A_{1 f r} \dot{x}-A_{3 r l} \dot{x}\right)}{2 C_{d}^{2} a_{12 f r}^{2}} \\
F_{d v r r} \approx \frac{\rho A_{1 r r}\left|A_{1 r r} \dot{x}-A_{3 f l} \dot{x}\right|\left(A_{1 r r} \dot{x}-A_{3 f l} \dot{x}\right)}{2 C_{d}^{2} a_{12 r r}^{2}}-\frac{\rho A_{3 r r}\left|A_{1 f l} \dot{x}-A_{3 r r} \dot{x}\right|\left(A_{1 f l} \dot{x}-A_{3 r r} \dot{x}\right)}{2 C_{d}^{2} a_{12 f l}^{2}}
\end{gathered}
$$


The damping forces in the roll mode are derived by letting $\dot{X}_{f l}=-\dot{X}_{f r}=\dot{X}_{r l}=-\dot{X}_{r r}=\dot{x}$. The pitch-mode damping forces of the front and rear struts of the X-coupled configuration $\mathrm{C} 1$ are also identical to those in the roll-mode. The resulting damping forces in the roll mode are given below:

$$
\begin{aligned}
& F_{d R f l} \approx \frac{\rho A_{1 f l}\left|A_{y l l} \dot{x}+A_{r z} \dot{x}\right|\left(A_{f l} \dot{x}+A_{r r} \dot{x}\right)}{2 C_{d}^{2} a_{12 l l}^{2}}+\frac{\rho A_{f l}\left|A_{3 r} \dot{x}+A_{f l} \dot{x}\right|_{3} A_{r r} \dot{x}+A_{1} A_{f l} \dot{x}}{2 C_{d}^{2} a_{\substack{r \\
b r 2}}^{2}} \\
& F_{d R f r} \approx \frac{\rho A_{1 f r}\left|A_{1 f r} \dot{x}+A_{3 r l} \dot{x}\right|\left(A_{1 f r} \dot{x}+A_{3 r l} \dot{x}\right)}{2 C_{d}^{2} a_{12 f r}^{2}}+\frac{\rho A_{3 f r}\left|A_{1 r l} \dot{x}+A_{3 f r} \dot{x}\right|\left(A_{1 r l} \dot{x}+A_{3 f r} \dot{x}\right)}{2 C_{d}^{2} a_{12 r l}^{2}}
\end{aligned}
$$

and

$$
\begin{aligned}
& F_{d R r l} \approx \frac{\rho A_{1 r l}\left|A_{1 r l} \dot{x}+A_{3 f r} \dot{x}\right|\left(A_{1 r l} \dot{x}+A_{3 f r} \dot{x}\right)}{2 C_{d}^{2} a_{12 r l}^{2}}+\frac{\rho A_{3 r l}\left|A_{1 f r} \dot{x}+A_{3 r l} \dot{x}\right|\left(A_{1 f r} \dot{x}+A_{3 r l} \dot{x}\right)}{2 C_{d}^{2} a_{12 f r}^{2}} \\
& F_{d R r r} \approx \frac{\rho A_{1 r r}\left|A_{1 r r} \dot{x}+A_{3 f l} \dot{x}\right|\left(A_{1 r r} \dot{x}+A_{3 f l} \dot{x}\right)}{2 C_{d}^{2} a_{12 r r}^{2}}+\frac{\rho A_{3 r r}\left|A_{1 f l} \dot{x}+A_{3 r r} \dot{x}\right|\left(A_{1 f l} \dot{x}+A_{3 r r} \dot{x}\right)}{2 C_{d}^{2} a_{12 f l}^{2}}
\end{aligned}
$$

It should also be noted that formulations for the warp-mode damping property of the $\mathrm{X}$-coupled configuration $\mathrm{C} 1$ are identical to those for the bounce-mode damping, described in Eq. (17).

The bounce-mode damping property of the unconnected suspension U1 is derived from the dissipative component of the strut forces, such that:

$$
F_{d v i} \approx \frac{\rho A_{2 i}^{3}|\dot{x}|(\dot{x})}{2 C_{d}^{2} a_{12 i}^{2}} \quad(i=f l \quad f r \quad r, l
$$


The roll-, pitch- and warp-mode damping properties of the unconnected suspension U1 can be derived from the above considering the absence of coupling among different suspension struts. The analytical formulations of damping properties of the other suspension configurations can also be derived in a similar manner using the generalized methodology described in [29].

\section{Results and Discussions}

The design parameters of the three fluidically-interconnected suspension configurations (C1 C3) and the unconnected suspension with and without anti-roll bars (U1bar and U1), are selected upon consideration of identical load carrying capacity for a freight vehicle, corresponding to a load distribution, $l_{f} /\left(l_{f}+l_{r}\right)=0.653$. The selected design parameters for all the five suspension configurations resulted in identical bounce-mode natural frequencies of the front and rear suspensions at the static design ride height, in the order of 1.5 Hz. The bleed and damping valves flow areas, and interconnecting pipe sizes were further chosen to achieve identical bounce-mode damping properties of the front and rear struts of all the selected configurations. The simulation results of the properties of the selected suspension configurations are presented and discussed below.

\subsection{Bounce Mode Properties}

Figures 15(a) and (b) present the bounce mode stiffness and damping properties of all the selected suspension configurations (C1 C3, U1 and U1bar), respectively. Under the in-phase bounce-mode excitations, all the suspensions yield identical front and rear suspension rates throughout the deflection range considered. The front suspension rates of 
all the configurations are lower than those of the rear suspension, which is attributed to the chosen load distribution. Both the front and rear suspensions exhibit progressively hardening property in compression, but softening effect in rebound, as evident in Fig. 15(a), which is attributed to the force-deflection property of the gas. The damping parameters were chosen to yield identical bounce-mode damping property of the frontand rear-axle suspensions, which is evident from the force-velocity relationships presented in Fig. 15(b). It should be noted that all the configurations are evaluated assuming symmetric damping in compression and rebound, while asymmetric damping properties can also be easily realized as described in Eq. (3). The flows through the valves are initiated when the strut velocity approaches or exceeds $0.08 \mathrm{~m} / \mathrm{s}$, and the valves become fully open at $1.5 \mathrm{~m} / \mathrm{s}$.

\section{FIGURE 15}

\subsection{Roll Mode Properties}

The variations in the roll stiffness of the front and rear suspensions of the selected configurations are presented in Figs. 16(a) and 16(b), respectively. The roll mode damping properties are presented in terms of force-velocity characteristics of the front and rear suspensions in Figs. 16(c) and 16(d), respectively. Unlike the bounce-mode properties, the three fluidically-coupled suspensions $\mathrm{C} 1 \sim \mathrm{C} 3$ exhibit significantly higher roll-mode properties, when compared to that of the uncoupled configuration U1. The additions of passive front and rear anti-roll bars can also yield an upward parallel shift of the effective roll stiffness of the unconnected suspension (U1bar), as it is shown in Figs. 16(a) and (b). Although the anti-roll bar yields static roll stiffness identical to those of the fluidically-coupled suspension configurations $\mathrm{C} 1 \sim \mathrm{C} 3$, the $\mathrm{X}$-coupled suspensions provide 
greater roll stiffness corresponding to higher deflections. Furthermore, the use of an anti-roll bar cannot augment the roll mode damping of the hydraulic X-coupled configurations, as shown in Figs. 16(c) and (d). The pneumatic interconnections (C2) also cannot help improve the roll-mode damping, while the hybrid interconnections (C3) yield considerable roll-mode damping gain compared to the U1bar and C2 configurations. The enhanced roll-mode damping properties of the $\mathrm{X}$-coupled $\mathrm{C} 1$ and $\mathrm{C} 3$ configurations would be beneficial for controlling the transient roll motions and roll stability limits during steering maneuvers [34].

\section{FIGURE 16}

Roll stiffness property of a suspension system strongly affects not only the roll stability of vehicles, but also the vehicle handling quality and directional stability limits $[1,3]$. Roll stiffness distribution is known to influence the handling balance (understeer/oversteer behavior). A vehicle with greater roll stiffness distributed over the front axle would exhibit greater understeer tendency. The static roll stiffness values of the front and rear suspensions can thus be tuned to help achieve a desirable understeer characteristic. A conventional nonlinear suspension system may induce considerable variations in the roll stiffness distribution over the range of vehicle roll deflections during steering, which may contribute to variations in the understeer coefficient. It is therefore highly desirable to investigate the dynamic roll stiffness distribution characteristics of a suspension with varying roll deflections.

The relative roll stiffness distribution ratio (RSDR), defined as the ratio of front suspension roll stiffness per unit sprung mass supported by the front suspension to that of the rear suspenion $\left(k_{R f} / m_{s f}\right) /\left(k_{R r} / m_{s r}\right)$, is used as a measure of the dynamic roll stiffness 
distribution, which may be applied to study the variations in understeer behavior under a directional maneuver. Figure 17 illustrates variations in the RSDR characteristics of all the five configurations as a function of the roll deflection. The results show constant roll stiffness distribution for the unconnected configuration U1 over the entire range of roll deflection considered, while the RSDR of the X-coupled configurations increases progressively with an increase in the roll deflection. Such progressive increase in RSDR with increasing roll deflection would indicate a greater understeer tendency during relatively high lateral-acceleration steering maneuvers. Configuration U1bar, using anti-roll bars, also yields higher RSDR, compared to the unconnected suspension U1 but considerably lower than the $\mathrm{X}$-coupled suspensions at higher roll deflections.

A few studies have suggested that an increase in the understeer coefficient with an increase in lateral acceleration (or during the nonlinear operating range of the tires) would be desirable for improved yaw or directional stability during tight or emergency type of steering maneuvers $[1,3,35,36]$. The results shown in Fig. 17 indicate that the X-coupled configurations ( $\mathrm{C} 1$ to $\mathrm{C} 3$ ) offer considerable potential in enhancing both the roll as well as directional stability limits of the road vehicles.

\section{FIGURE 17}

\subsection{Pitch Mode Properties}

The pitch stiffness properties of the five suspension configurations $(\mathrm{C} 1 \sim \mathrm{C} 3, \mathrm{U} 1$ and U1bar) are compared in Fig. 18. The results suggest that the X-coupled suspension configurations $\mathrm{C} 1 \sim \mathrm{C} 3$, yield considerably higher pitch stiffness than the uncoupled suspension U1, similar to the roll stiffness property. The suspension damping in the pitch 
mode varies identical to that in the roll mode, as seen in Figs. 16(c) and (d), for each of the fluidically-interconnected configurations. Particularly, the hydraulically-coupled suspension $\mathrm{C} 1$ yields considerably enhanced pitch-mode damping property due to additional flows through the couplings. The significantly higher pitch stiffness and pitch-mode damping of the hydraulically-coupled suspension could be beneficial in reducing the vehicle pitch motion during braking or acceleration maneuvers.

\section{FIGURE 18}

\subsection{Warp Mode Properties}

The roadholding and braking/traction performances of a vehicle are greatly influenced by the warp stiffness of the suspension. Figure 19 illustrates a comparison of the warp stiffness properties of the five suspension configurations, while the warp-mode damping was found to be identical to that in the bounce-mode for each configuration (Fig. 15(b)). The fluidically-interconnected suspension configurations yield warp stiffness identical to that of the unconnected suspension U1 over the entire range of the warp deflection considered, as in the case of bounce-mode stiffness. The use of anti-roll bars, however, tends to increase the effective suspension warp stiffness, due to the greater coupling in the roll and warp modes. This suggests that the fluidic-couplings do not alter the warp property of the suspension, while they yield significant gains in the roll as well as pitch mode properties. This finding is also consistent with the proposed VPI measure, described in Table 1.

FIGURE 19 
The fundamental suspension properties, illustrated in Figs. 15 to 19, clearly show that fluidic-coupling of the individual suspension struts could considerably increase the rolland pitch-mode stiffness and damping, while maintaining relatively low bounce- and warp-mode properties. This suggests that the selected fluidically-coupled suspension configurations could help realize greater decoupling between the roll/pitch and bounce/warp modes, so as to achieve an enhanced design compromise among the four vehicle vibration-modes. The higher roll- and pitch-mode stiffness values were also reflected in the VPI values presented in Table 1, which further anticipated minimal changes in the bounce- and warp-stiffness properties compared to those of the unconnected configuration. This supports the proposed simplified VPI measure for preliminary assessments and feasibility analyses of alternative full-vehicle interconnected suspension configurations.

The fluidically-coupled hydro-pneumatic suspensions also offer greater design flexibility, apart from the enhanced stiffness and damping properties. The suspension properties could be conveniently varied through variations in the strut geometry. It has been established that variations in the annular piston areas $\left(A_{3}\right)$ of the struts yields the greatest variations in stiffness properties of suspensions connected either in the roll- or the pitch-plane, without affecting the load carrying capacity and the operating pressure requirement [14-16]. In this study, the influence of variations in the annular piston area $\left(A_{3 f}\right)$ of the front struts on the resulting stiffness properties of the hydraulically-coupled suspension configuration $\mathrm{C} 1$ is investigated, as an example. The area is varied by $\pm 20 \%$ about the nominal value, while maintaining identical load carrying capacity and static gas pressure. The resulting variations in the front and rear suspension rates are presented in Figs. 20(a) and 20(b), respectively. The variations in $A_{3 f}$ cause only minimal changes in the front and rear 
suspension rates in the rebound mode, while the effect is relatively large on both the suspension rates in the compression mode. It is further observed that an increase in $A_{3 f}$ yields a higher front suspension rate, but a lower rear suspension rate, which is attributed to the coupling effect between the front and rear suspension struts.

The influence of variations in $A_{3 f}$ on the front and rear suspension roll stiffness properties, and the roll stiffness distribution ratio (RSDR) are presented in Figs. 21 and 22, respectively. An increase in $A_{3 f}$ not only yields higher roll mode stiffness of the front and rear suspensions, but also a higher RSDR of the full-vehicle hydraulically-coupled suspension system. An increase in $A_{3 f}$ would thus yield a more understeer tendency with increasing roll deflection of the suspension.

FIGURE 20

FIGURE 21

\section{FIGURE 22}

Figures 23(a) and 23(b) present the influence of variations in $A_{3 f}$ on the pitch and warp stiffness of the hydraulically-coupled suspension $\mathrm{C} 1$, respectively. The results show that an increase in $A_{3 f}$ yields higher suspension pitch stiffness, suggesting a stronger coupling between the front and rear suspensions. A decrease in $A_{3 f}$, however, would reduce the warp stiffness of the hydraulically-coupled suspension $\mathrm{C} 1$.

\section{FIGURE 23}




\section{Conclusions}

A number of full-vehicle interconnected hydro-pneumatic suspension configurations were conceived and analyzed in terms of their feasibility and anticipated properties in the bounce-, roll-, pitch- and warp-modes. A simplified measure, referred to as vehicle property index (VPI), was proposed for preliminary assessments of possible fluidically-coupled suspension configurations. It is shown that different fluidic couplings among the four suspension struts could yield significantly different stiffness and damping properties. The static and dynamic forces developed by the struts of the selected fluidically-coupled suspensions were analyzed to derive suspension stiffness and damping properties in the four fundamental vibration-modes, namely bounce, roll, pitch and warp. The simulation results showed that the fluidically X-coupled suspension configurations could increase the roll- and pitch-mode stiffness and damping substantially, without affecting the bounce- and warp-mode properties. Such enhanced decoupling of the roll/pitch from the bounce/warp modes would be beneficial in realizing an improved design compromise among various vehicle performance measures under complex driving conditions. It is further concluded that X-coupled suspension configurations employing hydro-pneumatic struts offer greater design flexibility. Particularly high sensitivity of the stiffness properties to variations in the piston annual area was demonstrated, which could be beneficial in realizing greater roll and pitch-mode stiffness with negligible effect on the load carrying capacity and the operating pressure requirement.

\section{References}


1. Cole, D.J., 2001, 'Fundamental issues in suspension design for heavy road vehicles', Vehicle System Dynamics, 35, p.319-360.

2. Ahmadian, M. and Marjoram, R. H., 1989, 'Effects of Passive and Semi-active Suspensions on Body and Wheelhop Control', SAE Journal of Commercial Vehicles, 98, p.596-604.

3. Wong, J.Y., 2001, 'Theory of ground vehicles', $3^{\text {rd }}$ Edition, John Wiley \& Sons, Inc., USA.

4. Crolla, D.A., 1996, 'Vehicle dynamics - theory into practice', Journal of Automobile Engineering, 210, p.83-94.

5. Wilde, J.R., Heydinger, G.J., Guenther, D.A., Mallin, T. and Devenish, A.M., 2005, 'Experimental evaluation of fishhook maneuver performance of a kinetic suspension system', SAE paper 2005-01-0392.

6. Woods, D.E. and Jawad, B.A., 1999, 'Numerical design of racecar suspension parameters', SAE paper 1999-01-2257.

7. Zapletal, E., 2000, 'Balanced suspension', SAE paper 2000-01-3572.

8. Buj, J.F., 2002, 'Integral suspension system for motor vehicles based on passive components', SAE paper 2002-01-3105.

9. El-Demerdash, S.M., Selim, A.M. and Crolla, D.A., 1999, "Vehicle body attitude control using an electronically controlled active suspension", SAE paper 1999-01-0724.

10. Dahlberg, E., 1999, 'Yaw instability due to longitudinal load transfer during braking in a curve', SAE paper 1999-01-2952. 
11. Sharp, R.S., 1999, 'Vehicle dynamics and the judgment of quality', Vehicle Performance, Edited by Pauwelussen, J.P., Swets \& Zeitlinger Publishers, Lisse, the Netherlands, p.87-96.

12. Williams, R.A., 1997, 'Automotive active suspensions. Part 2: practical considerations', Journal of Automobile Engineering, 211, p.427-444.

13. Rajamani, R., 2006, 'Vehicle dynamics and control', Springer, NY, USA.

14. Cao, D., Rakheja, S. and Su, C.-Y., 2006, 'A generalized model of a class of interconnected hydro-pneumatic suspensions and analysis of pitch properties', Proc. of ASME Int. Mech. Engineering Congress, IMECE2006-13961, Chicago, USA.

15. Cao, D., Rakheja, S. and Su, C.-Y., 2007, 'Pitch Attitude Control and Braking Performance Analysis of Heavy Vehicles with Interconnected Suspensions', SAE paper 2007-01-1347.

16. Cao, D., Rakheja, S. and Su, C.-Y., 'Dynamic analyses of roll plane interconnected hydro-pneumatic suspension systems', International Journal of Vehicle Design. (In press)

17. Felez, J. and Vera, C., 1987, 'Bond graph assisted models for hydro-pneumatic suspensions in crane vehicles', Vehicle System Dynamics, 16, p.313-332.

18. Rakheja, S., Liu, P., Ahmed, A.K.W. and Su, H., 1993, 'Analysis of an interlinked hydro-pneumatic suspension', Proc. of ASME Winter Annual Meeting, New Orleans, USA.

19. Horton, D.N.L. and Crolla, D.A., 1986, 'Theoretical analysis of a semi-active suspension fitted to an off-road vehicle', Vehicle System Dynamics, 15, p.351-372. 
20. Feury, M., Halle, N., Simon, G. and Stinson, M., 2001, 'Future heavy tactical truck', SAE paper 2001-01-0889.

21. Gunter, D., Bylsma, W., Letherwood, M., Dennis, S., Argeropoulos, K., Teschendorf, D. and Gorsich, D., 2005, 'Using 3D multi-body simulation to evaluate future truck technologies', SAE paper 2005-01-0934.

22. Giliomee, C.L., 2003, 'Analysis of a four state switchable hydro-pneumatic spring and damper system', Master thesis, University of Pretoria, South Africa.

23. Su, H., 1990, 'An investigation of vibration isolation systems using active, semi-active and tunable passive mechanisms with applications to vehicle suspensions', $\mathrm{PhD}$ thesis, Concordia University, Canada.

24. Liu, P., 1994, 'An analytical study of ride and handling performance of an interconnected vehicle suspension', Master thesis, Concordia University, Canada.

25. Chaudhary, S., 1998, 'Ride and roll performance analysis of a vehicle with spring loaded interconnected hydro-pneumatic suspension', Master thesis, Concordia University, Canada.

26. Wilde, J.R., Heydinger, G.J. and Guenther, D.A., 2006, 'ADAMS simulation of ride and handling performance of kinetic suspension system', SAE paper 2006-01-1972.

27. Cao, D., Rakheja, S. and Su, C.-Y., 2008, 'Pitch plane analysis of a twin-gas-chamber strut suspension', Journal of Automobile Engineering. (In press)

28. Cao, D., Rakheja, S. and Su, C.-Y., 2007, 'Roll plane analysis of a hydro-pneumatic suspension with twin-gas-chamber struts', International Journal of Heavy Vehicle Systems, 14, p.355-375. 
29. Cao, D., 2008, 'Theoretical analyses of roll- and pitch-coupled hydro-pneumatic strut suspensions', PhD thesis, Concordia University, Canada.

30. Dixon, J.C., 1999, 'The shock absorber handbook', SAE Inc., USA.

31. Ahmadian, M. and Ahn, Y.K., 2003, 'On-vehicle evaluation of heavy truck suspension kinematics', SAE paper 2003-01-3394.

32. Sutton, T., Pope, R., Brandewie, K. and Henry, J., 2001, 'International truck and engine high performance vehicle driveability - ride and handling', SAE paper 2001-01-2787.

33. Hac, A., 2002, 'Influence of active chassis systems on vehicle propensity to maneuver-induced rollovers', SAE paper 2002-01-0967.

34. Winkler, C., 2000, 'Rollover of heavy commercial vehicles', UMTRI Research Review, 31, The University of Michigan, USA.

35. Sampson, D.J.M. and Cebon, D., 2003, 'Achievable roll stability of heavy road vehicles', Journal of Automobile Engineering, 217, p. 269-297.

36. Metz, L.D., 2004, 'What constitutes good handling?', SAE paper 2004-01-3532.

37. Milliken, W.F. and Milliken, D.L., 2002, 'Chassis design: principles and analysis', SAE Inc., USA. 
Table 1: Comparisons of vehicle property indeces (VPIs) of selected suspension configurations for applications in heavy vehicles.

\begin{tabular}{|c|c|c|c|c|c|}
\hline \multirow{2}{*}{$\begin{array}{l}\text { Suspension } \\
\text { configuration }\end{array}$} & \multicolumn{4}{|c|}{ Static stiffness scaling } & \multirow{2}{*}{ VPI } \\
\hline & Bounce & Roll & Pitch & Warp & \\
\hline U1-U4 & 3 & 3 & 3 & 3 & 1.4 \\
\hline U1bar-U4bar & 3 & 5 & 3 & 4 & 0.5 \\
\hline $\mathrm{C} 1$ & 3 & 5 & 5 & 3 & 0.2 \\
\hline $\mathrm{C} 2$ & 3 & 5 & 5 & 3 & 0.2 \\
\hline C3 & 3 & 5 & 5 & 3 & 0.2 \\
\hline $\mathrm{C} 4$ & 3 & 5 & 5 & 3 & 0.2 \\
\hline C5 & 3 & 5 & 3 & 4 & 0.5 \\
\hline C6 & 3 & 5 & 3 & 4 & 0.5 \\
\hline $\mathrm{C} 7$ & 3 & 5 & 3 & 4 & 0.5 \\
\hline $\mathrm{C} 8$ & 3 & 5 & 3 & 4 & 0.5 \\
\hline C9 & 3 & 3 & 1 & 2 & 1.5 \\
\hline $\mathrm{C} 10$ & 3 & 3 & 1 & 2 & 1.5 \\
\hline $\mathrm{C} 11$ & 3 & 3 & 1 & 2 & 1.5 \\
\hline $\mathrm{C} 12$ & 3 & 3 & 1 & 2 & 1.5 \\
\hline C13 & 3 & 1 & 1 & 1 & 2.4 \\
\hline C14 & 3 & 1 & 1 & 1 & 2.4 \\
\hline C15 & 3 & 5 & 4 & 4.5 & 0.45 \\
\hline C16 & 3 & 5 & 4 & 4.5 & 0.45 \\
\hline C17 & 3 & 3 & 5 & 4 & 1.3 \\
\hline C18 & 3 & 3 & 5 & 4 & 1.3 \\
\hline
\end{tabular}


Fig. 1: Schematics of two novel hydro-pneumatic strut designs: (a) single-gas-chamber strut $B$; and (b) twin-gas-chamber strut $A$.

Fig. 2: Representation of an interconnection configuration in the vehicle roll- and pitch-plane arrangement.

Fig. 3: Two feasible 4-DOF full-vehicle interconnected suspensions involving single-gas-chamber struts with hydraulic couplings.

Fig. 4: Two feasible 4-DOF interconnected suspensions involving twin-gas-chamber struts with pneumatic couplings.

Fig. 5: Two feasible 4-DOF interconnected suspensions involving single- and twin-gas chamber struts with hydraulic and pneumatic interconnections.

Fig. 6: A 6-DOF interconnected suspension system using only twin-gas-chamber struts with pneumatic interconnections.

Fig. 7: Full-vehicle unconnected hydro-pneumatic suspension configurations (U1, U2, U3 and U4) using single- and twin-gas chamber struts.

Fig. 8: Four-DOF full-vehicle interconnected hydro-pneumatic suspension configurations involving $\mathrm{X}$-couplings $(\mathrm{C} 1, \mathrm{C} 2, \mathrm{C} 3$ and $\mathrm{C} 4)$.

Fig. 9: Four-DOF full-vehicle interconnected suspension configurations involving fluidic couplings in the roll-plane (C5, C6, C7 and C8).

Fig. 10: Full-vehicle interconnected suspension configurations involving pneumatic couplings in the pitch-plane (C9, C10, C11 and C12).

Fig. 11: Six-DOF interconnected full-vehicle suspension configurations based on twin-gas chamber struts with pneumatic X-couplings (C13 and C14).

Fig. 12: Four-DOF interconnected suspension configurations employing single- and twin-gas-chamber struts with roll- and pitch-plane couplings (C15 and C16).

Fig. 13: Four-DOF interconnected configurations based on single- and twin-gas-chamber struts with pitch-plane couplings.

Fig. 14: Simplified model representation of the struts with fixed sprung mass for evaluating properties of the interconnected and unconnected suspension configurations.

Fig. 15: Bounce-mode properties of the front and rear suspensions of the selected configurations $(\mathrm{C} 1, \mathrm{C} 2, \mathrm{C} 3, \mathrm{U} 1$ and $\mathrm{U} 1 \mathrm{bar})$ : (a) suspension rate; and (b) damping.

Fig. 16: Roll-mode properties of the unconnected and X-coupled suspension configurations: (a) front suspension roll stiffness; (b) rear suspension roll stiffness; (c) front suspension roll-mode damping; and (d) rear suspension roll-mode damping.

Fig. 17: Comparisons of the roll stiffness distribution ratio (RSDR) characteristics of the unconnected and X-coupled full-vehicle suspension configurations.

Fig. 18: Variations in pitch stiffness of the full-vehicle unconnected (U1 and U1bar) and 
$\mathrm{X}$-coupled (C1, C2 and C3) suspension configurations.

Fig. 19: Variations in the warp stiffness properties of the full-vehicle unconnected (U1 and U1bar) and X-coupled (C1, C2 and C3) suspension configurations.

Fig. 20: Influence of variations in the front-strut annular area $A_{3 f}$ on the bounce-mode stiffness of the hydraulically-coupled suspension C1: (a) front suspension rate; and (b) rear suspension rate.

Fig. 21: Influence of variations in the front-strut annular area $A_{3 f}$ on the roll-mode stiffness of the hydraulically-coupled suspension C1: (a) front suspension roll stiffness; and (b) rear suspension roll stiffness.

Fig. 22: Influence of variations in $A_{3 f}$ on the roll stiffness distribution ratio (RSDR) of the hydraulically-coupled suspension $\mathrm{C} 1$.

Fig. 23: Influence of variations in $A_{3 f}$ on: (a) pitch stiffness; and (b) warp stiffness of the hydraulically-coupled suspension $\mathrm{C} 1$. 


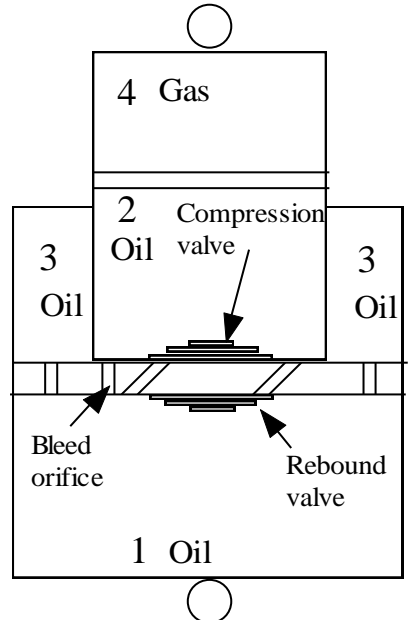

(a)

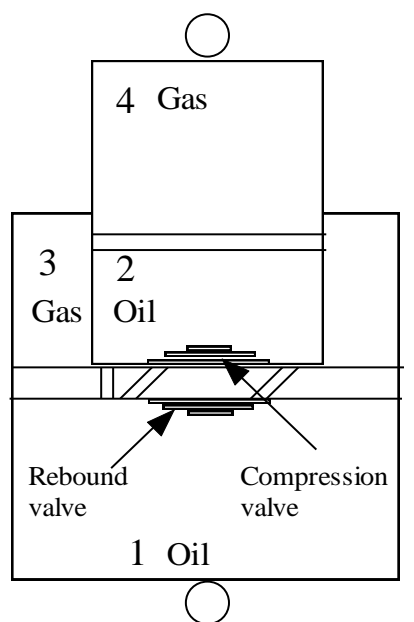

(b)

Fig. 1: Schematics of two novel hydro-pneumatic strut designs: (a) single-gas-chamber strut $B$; and (b) twin-gas-chamber strut $A$.

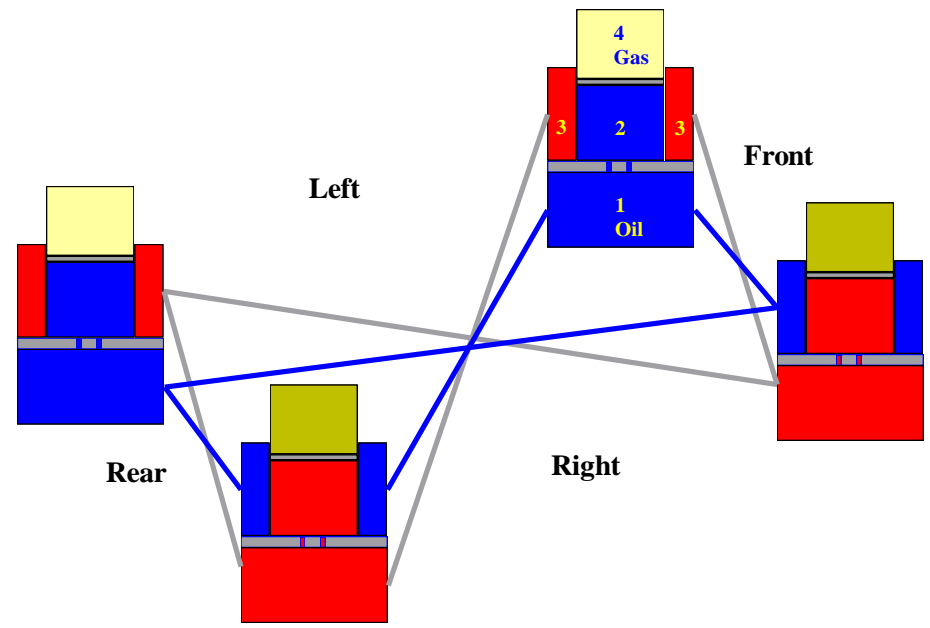

Fig. 2: Representation of an interconnection configuration in the vehicle roll- and pitch-plane arrangement.

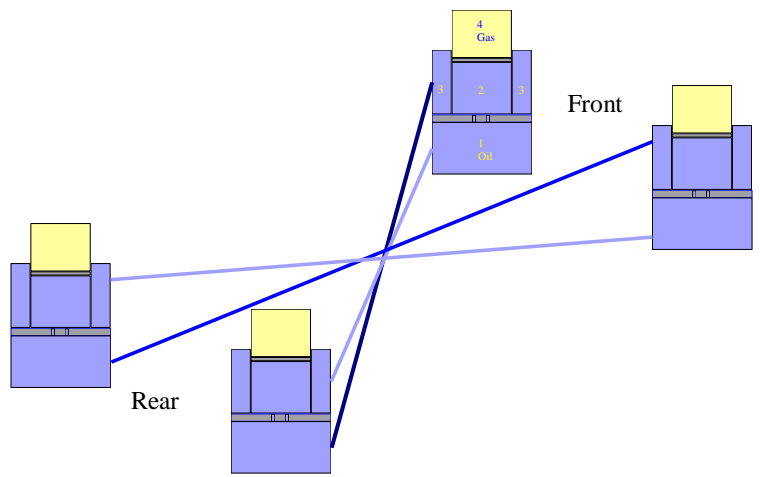

(a)

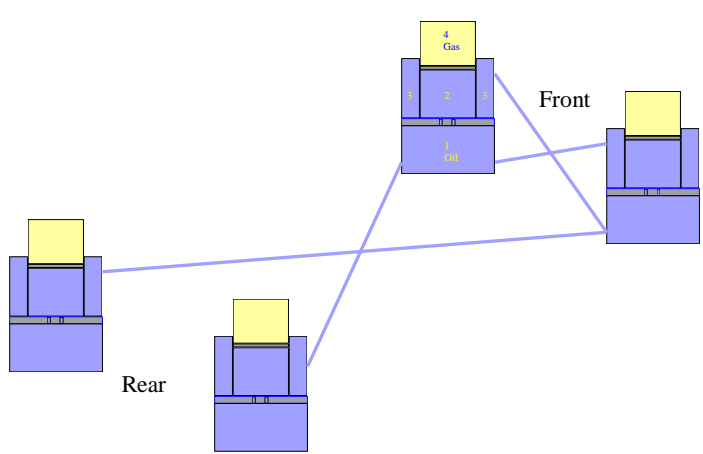

(b)

Fig. 3: Two feasible 4-DOF full-vehicle interconnected suspensions involving single-gas-chamber struts with hydraulic couplings. 


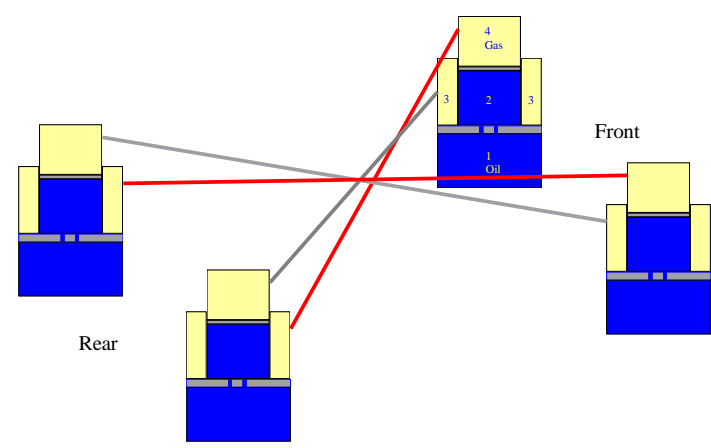

(a)

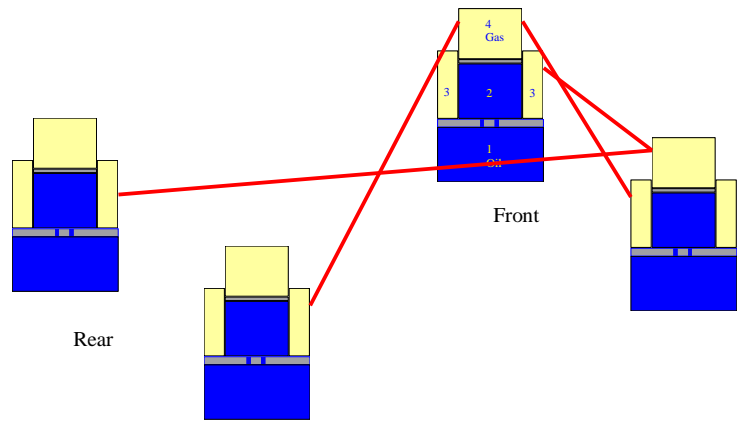

(b)

Fig. 4: Two feasible 4-DOF interconnected suspensions involving twin-gas-chamber struts with pneumatic couplings.

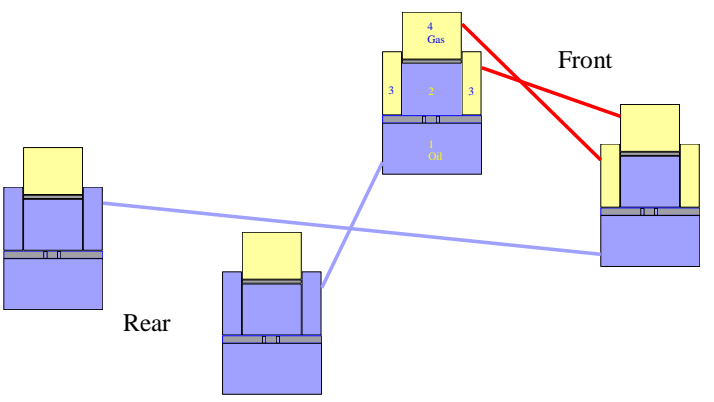

(a)

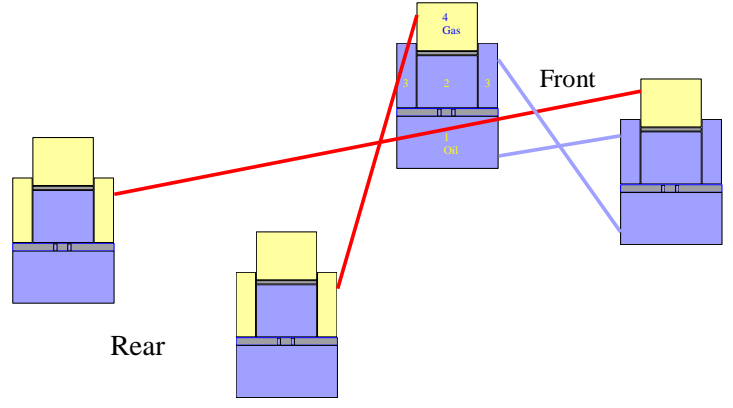

(b)

Fig. 5: Two feasible 4-DOF interconnected suspensions involving single- and twin-gas chamber struts with hydraulic and pneumatic interconnections.

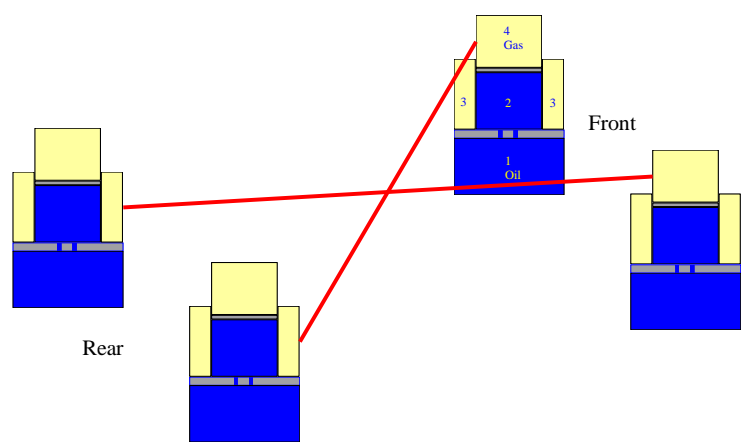

Fig. 6: A 6-DOF interconnected suspension system using only twin-gas-chamber struts with pneumatic interconnections. 

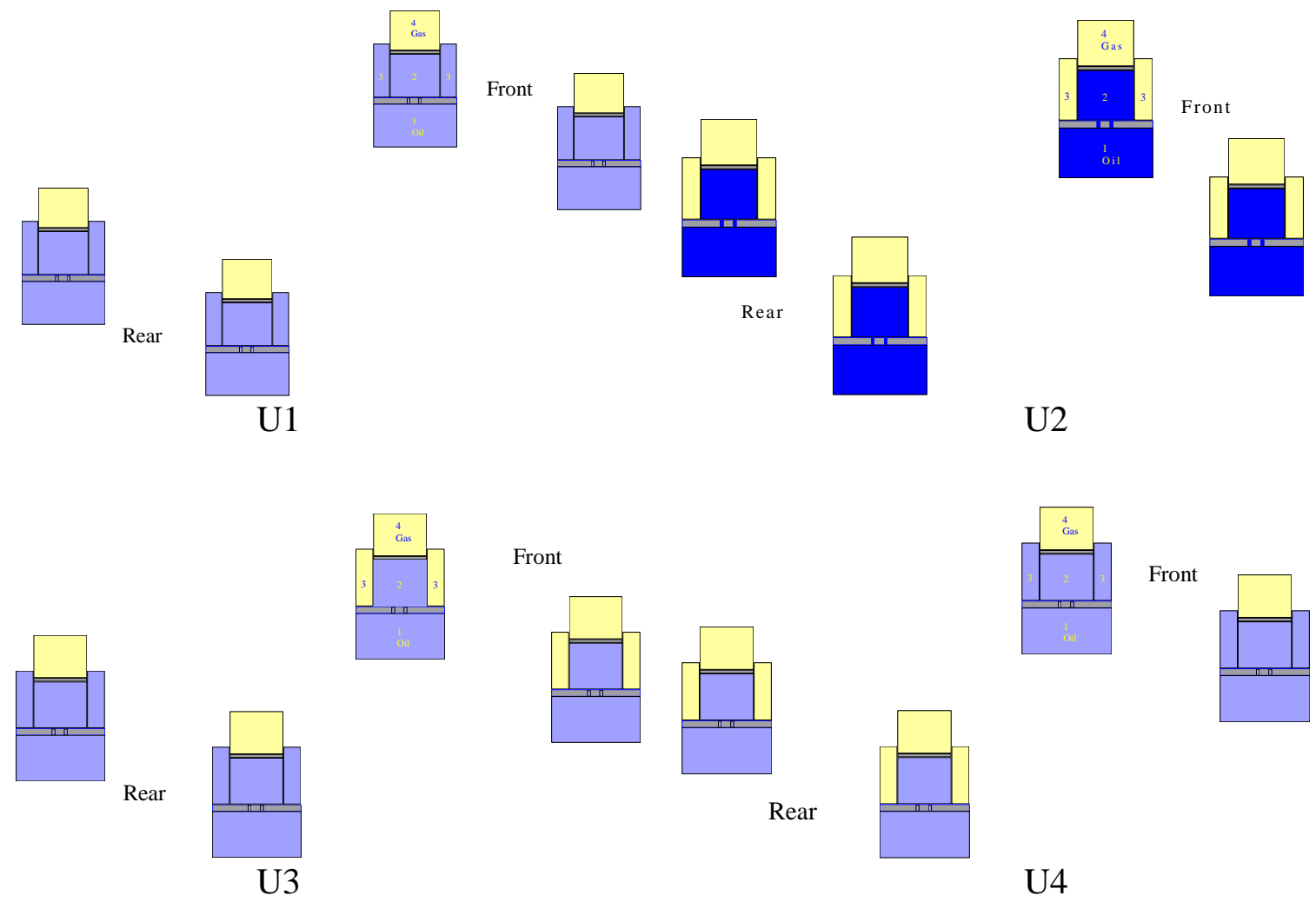

Fig. 7: Full-vehicle unconnected hydro-pneumatic suspension configurations (U1, U2, U3 and U4) using single- and twin-gas chamber struts.
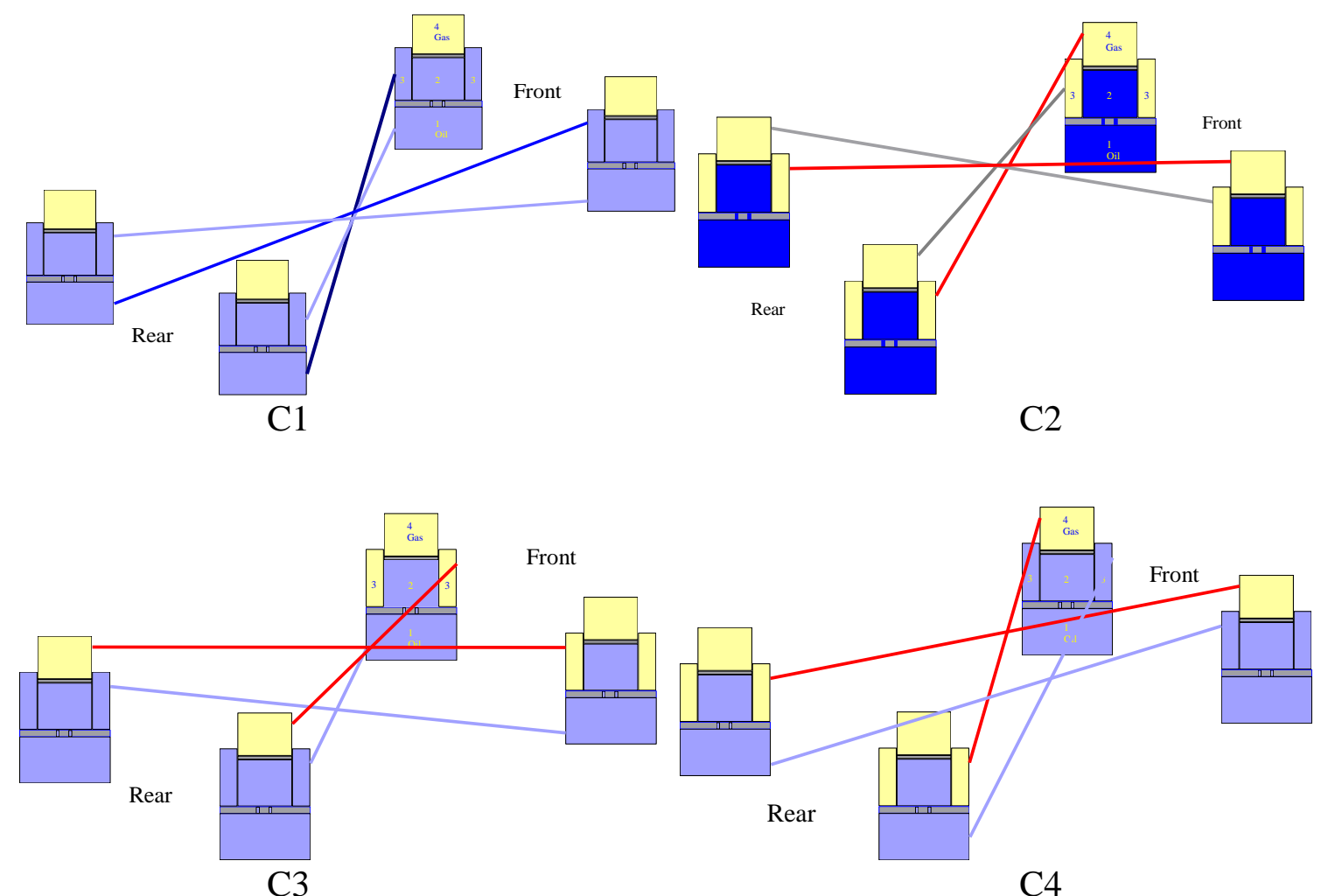

Fig. 8: Four-DOF full-vehicle interconnected hydro-pneumatic suspension configurations involving $\mathrm{X}$-couplings $(\mathrm{C} 1, \mathrm{C} 2, \mathrm{C} 3$ and $\mathrm{C} 4)$. 

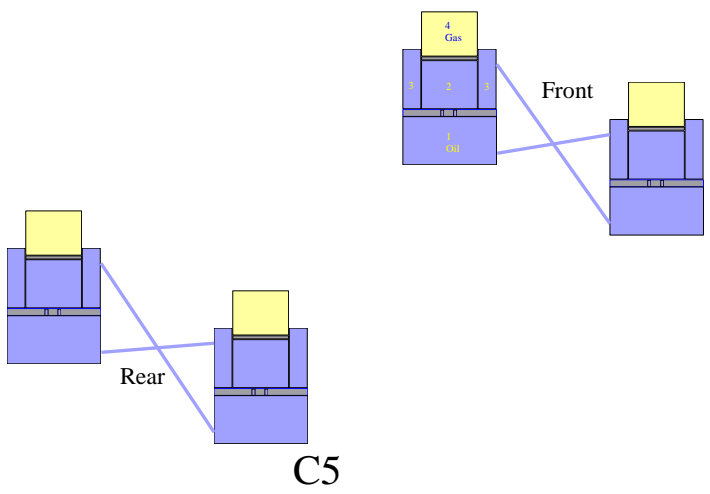

C5
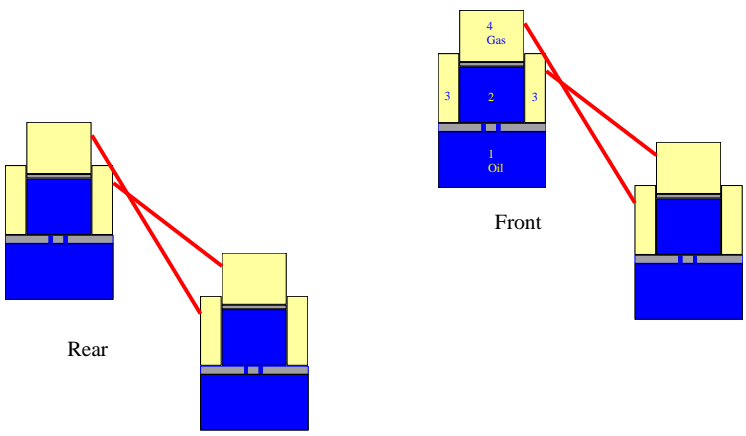

C6
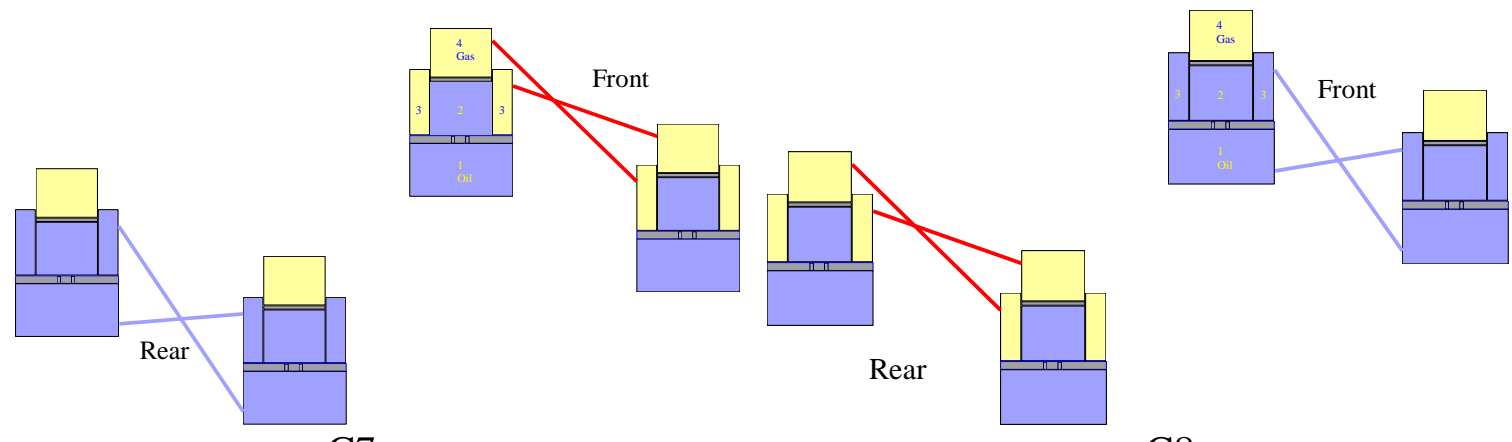

C7

Fig. 9: Four-DOF full-vehicle interconnected suspension configurations involving fluidic couplings in the roll-plane (C5, C6, C7 and C8).
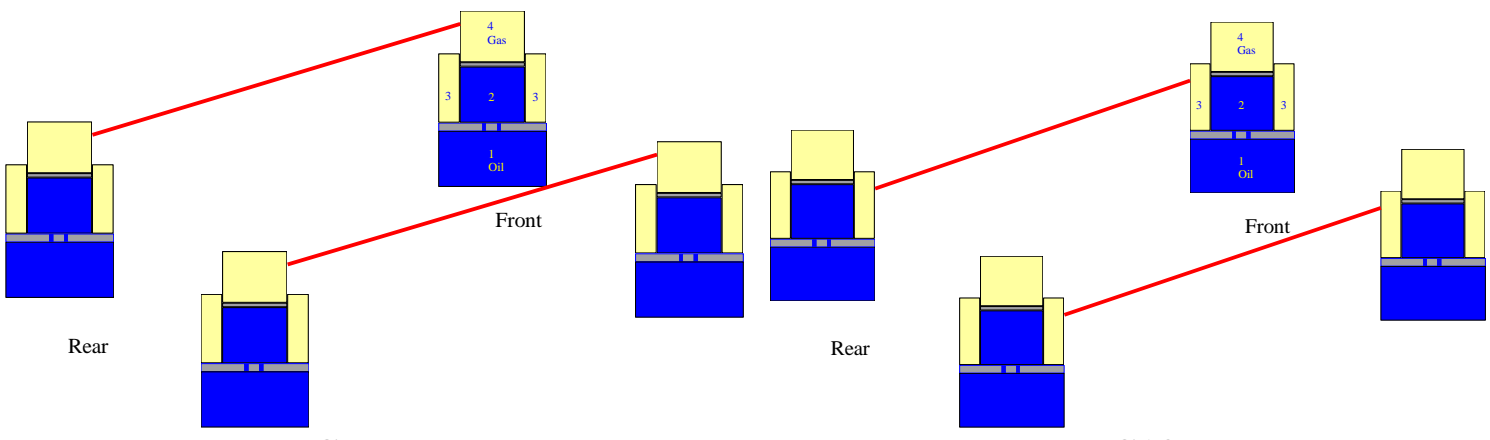

C9

$\mathrm{C} 10$
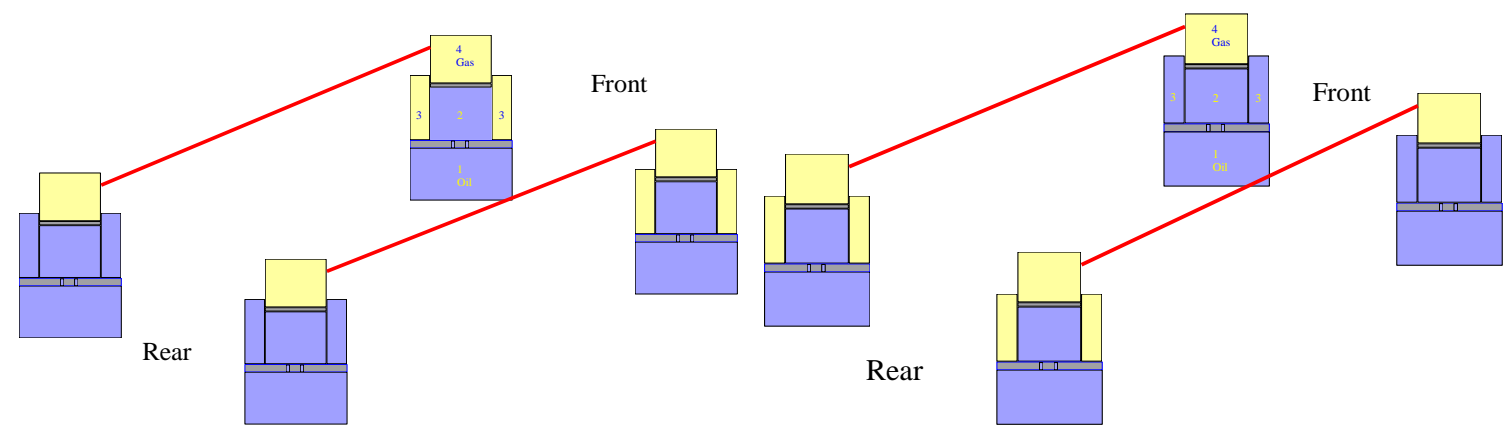

$\mathrm{C} 11$

C12

Fig. 10: Full-vehicle interconnected suspension configurations involving pneumatic couplings in the pitch-plane $(\mathrm{C} 9, \mathrm{C} 10, \mathrm{C} 11$ and $\mathrm{C} 12)$. 


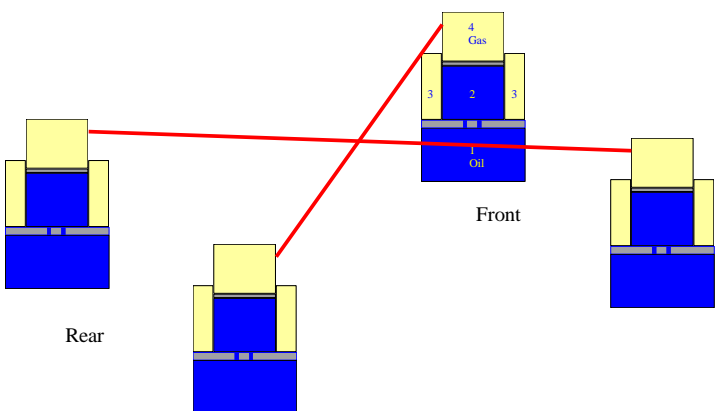

$\mathrm{C} 13$

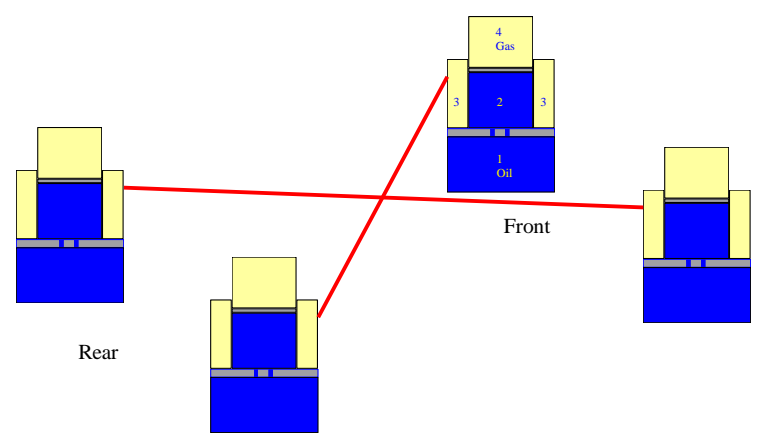

C14

Fig. 11: Six-DOF interconnected full-vehicle suspension configurations based on twin-gas chamber struts with pneumatic X-couplings (C13 and C14).

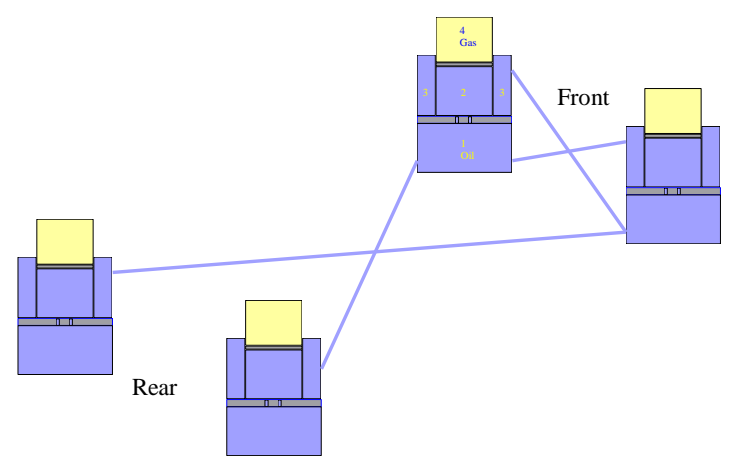

C15

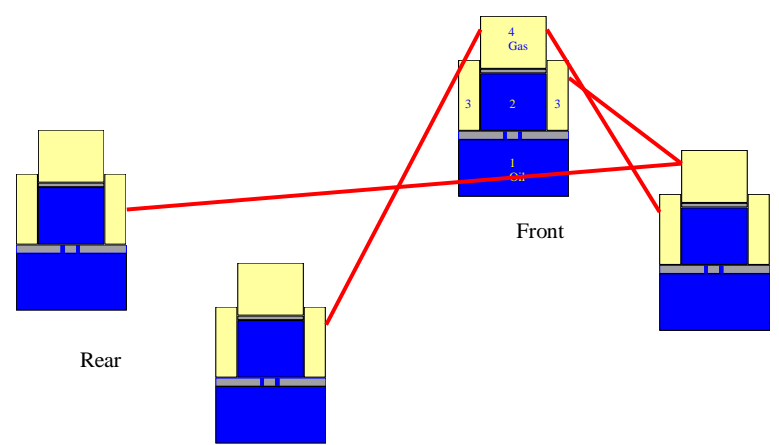

C16

Fig. 12: Four-DOF interconnected suspension configurations employing single- and twin-gas-chamber struts with roll- and pitch-plane couplings (C15 and C16).

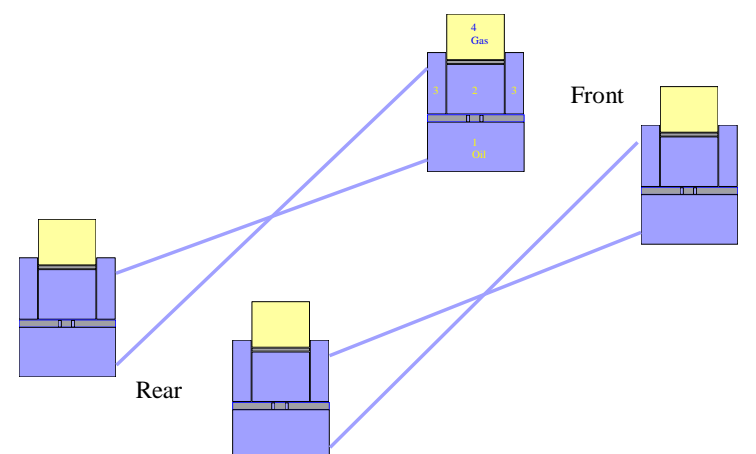

$\mathrm{C} 17$

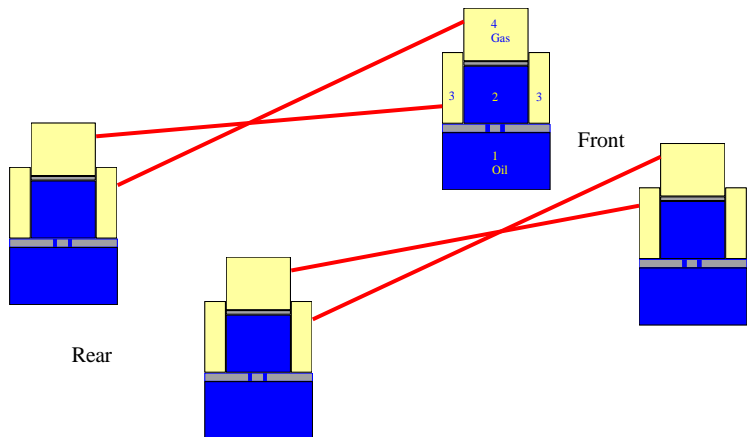

C18

Fig. 13: Four-DOF interconnected configurations based on single- and twin-gas-chamber struts with pitch-plane couplings. 


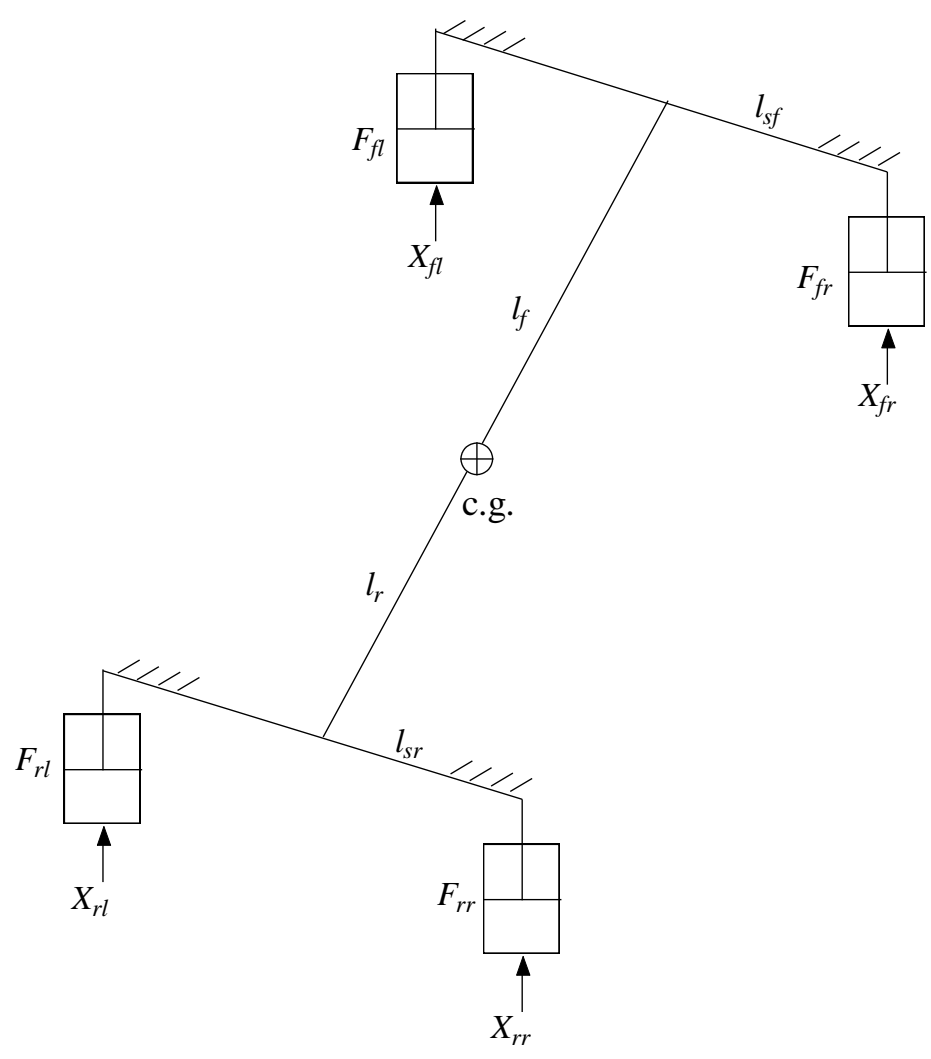

Fig. 14: Simplified model representation of the struts with fixed sprung mass for evaluating properties of the interconnected and unconnected suspension configurations.

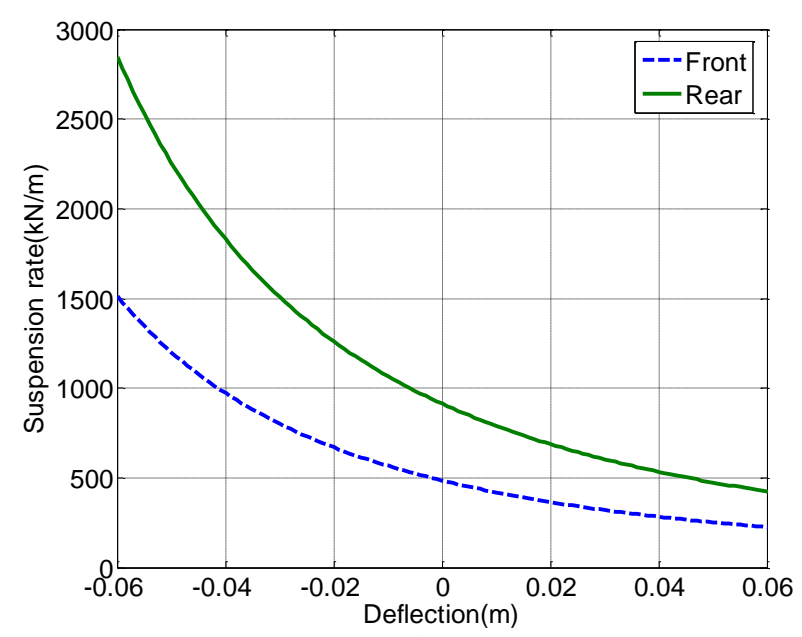

(a)

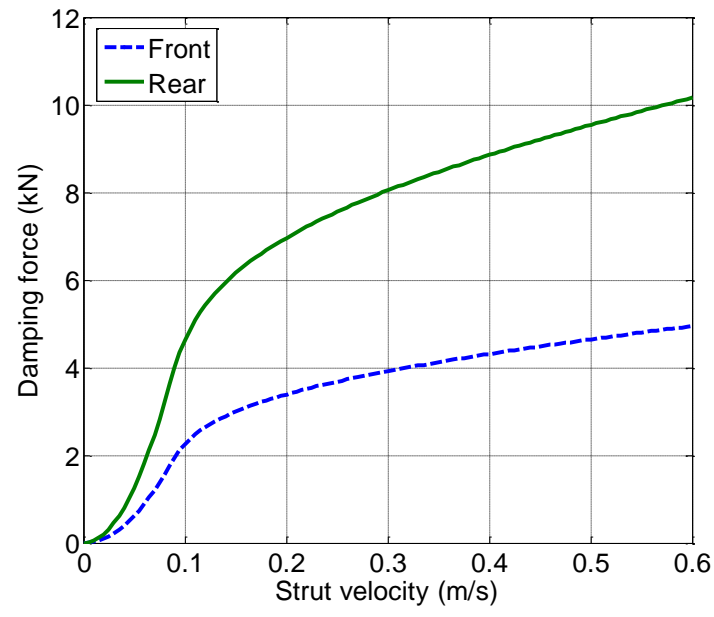

(b)

Fig. 15: Bounce-mode properties of the front and rear suspensions of the selected configurations ( $\mathrm{C} 1, \mathrm{C} 2, \mathrm{C} 3, \mathrm{U} 1$ and $\mathrm{U} 1 \mathrm{bar})$ : (a) suspension rate; and (b) damping. 


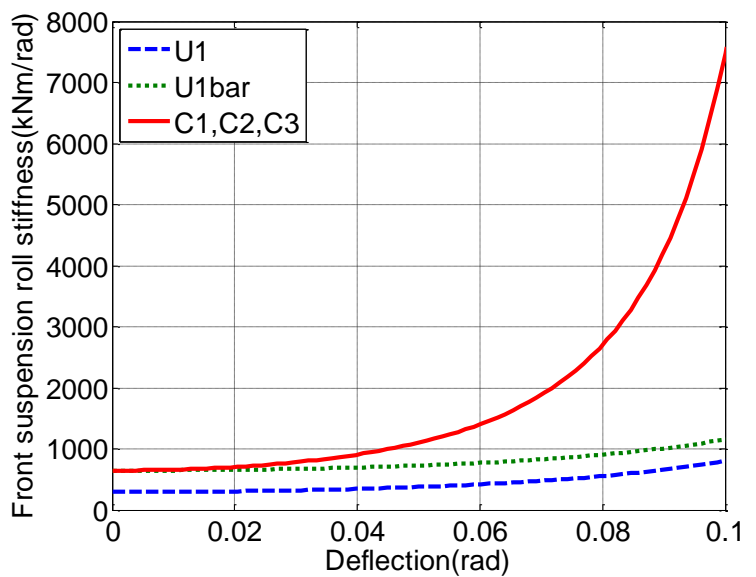

(a)

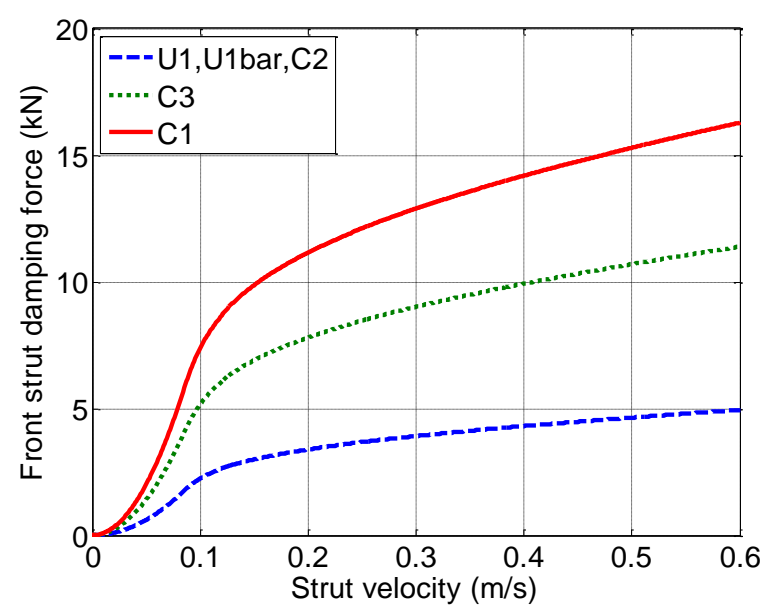

(c)

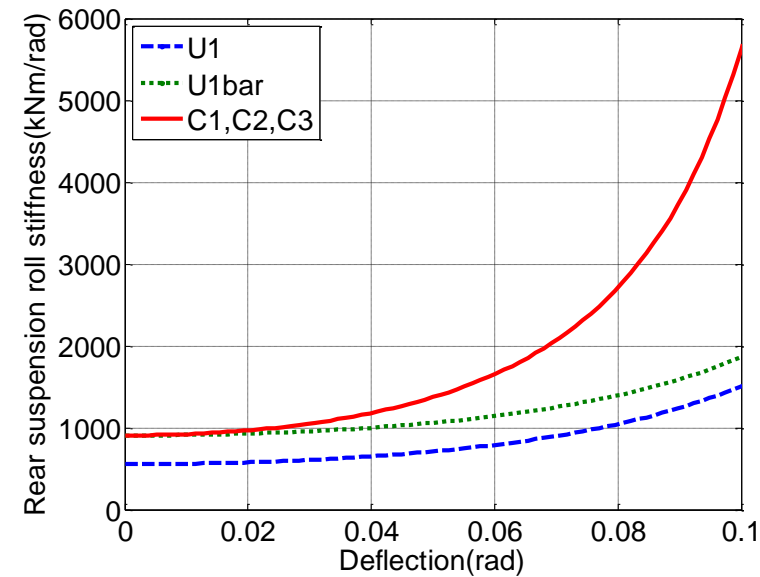

(b)

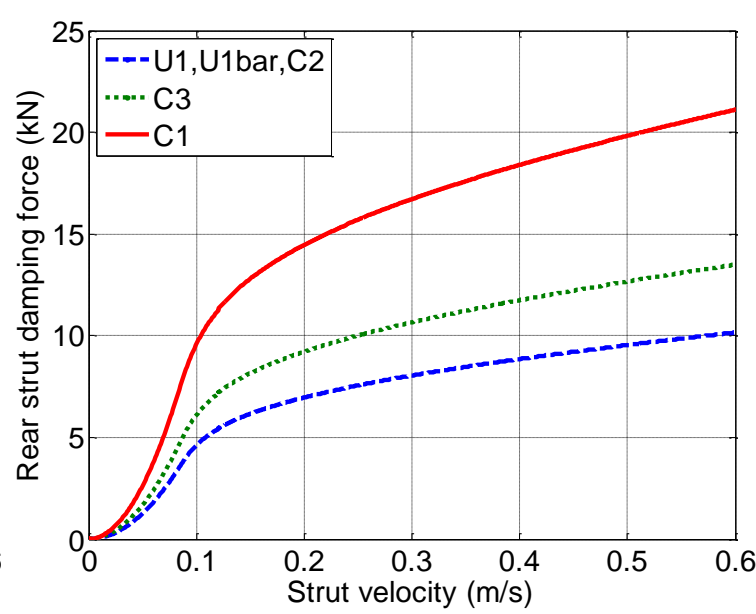

(d)

Fig. 16: Roll-mode properties of the unconnected and X-coupled suspension configurations: (a) front suspension roll stiffness; (b) rear suspension roll stiffness; (c) front suspension roll-mode damping; and (d) rear suspension roll-mode damping.

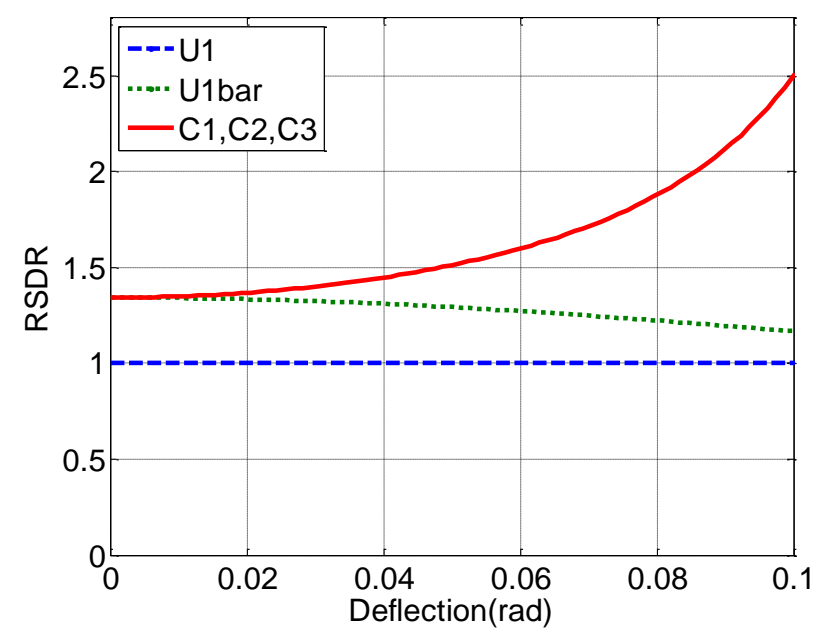

Fig. 17: Comparisons of the roll stiffness distribution ratio (RSDR) characteristics of the unconnected and X-coupled full-vehicle suspension configurations. 


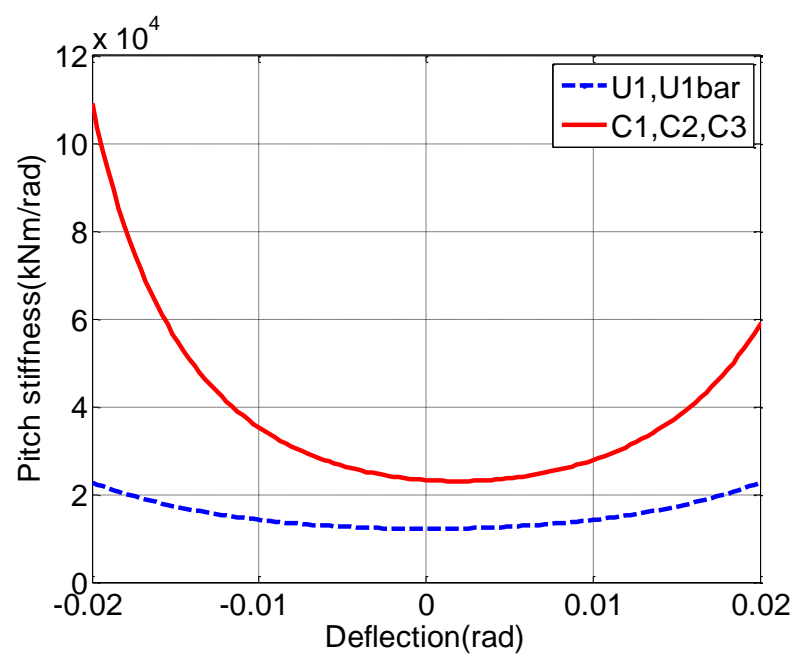

Fig. 18: Variations in pitch stiffness of the full-vehicle unconnected (U1 and U1bar) and $\mathrm{X}$-coupled (C1, C2 and C3) suspension configurations.

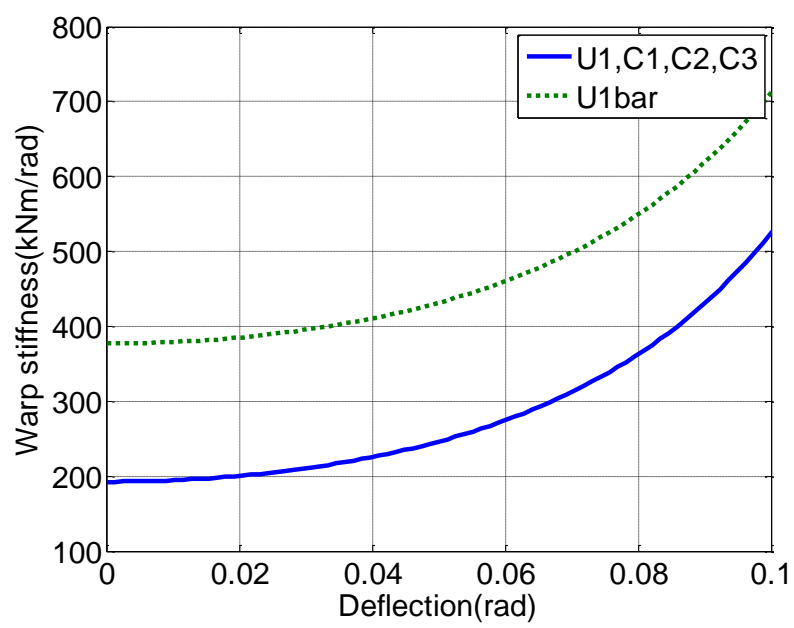

Fig. 19: Variations in the warp stiffness properties of the full-vehicle unconnected (U1 and $\mathrm{U} 1$ bar) and $\mathrm{X}$-coupled (C1, C2 and C3) suspension configurations.

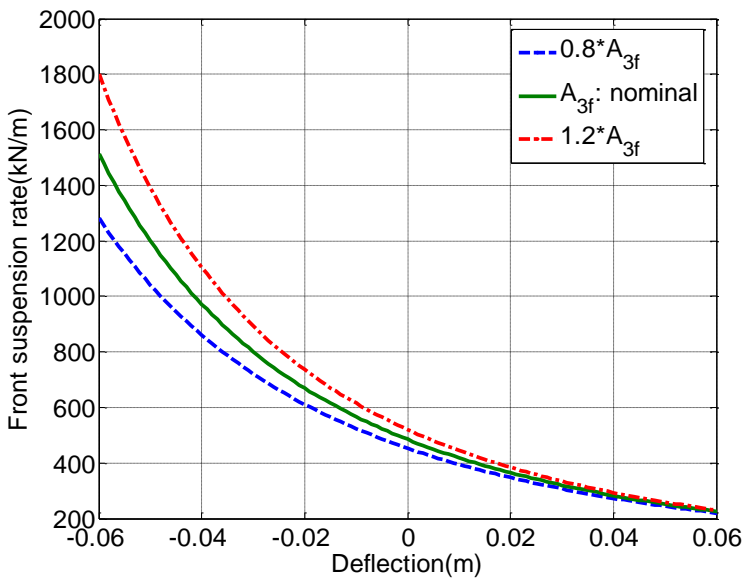

(a)

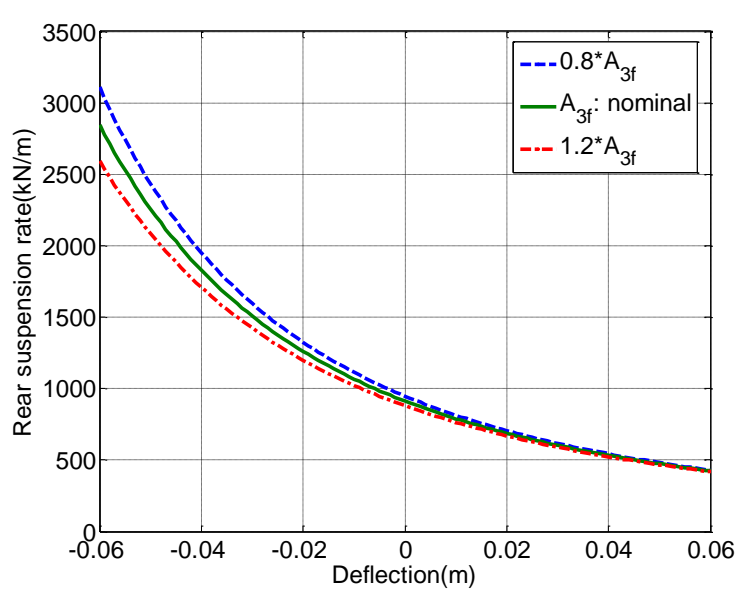

(b)

Fig. 20: Influence of variations in the front-strut annular area $A_{3 f}$ on the bounce-mode stiffness of the hydraulically-coupled suspension C1: (a) front suspension rate; and (b) rear suspension rate. 


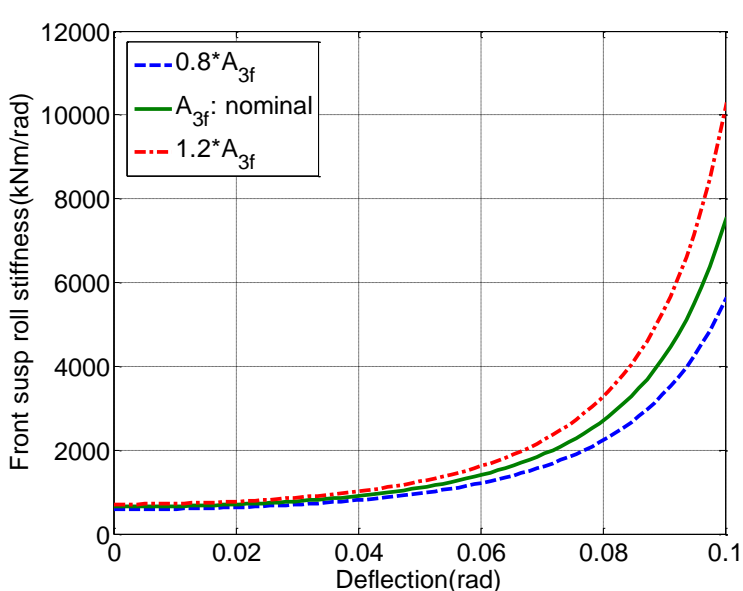

(a)

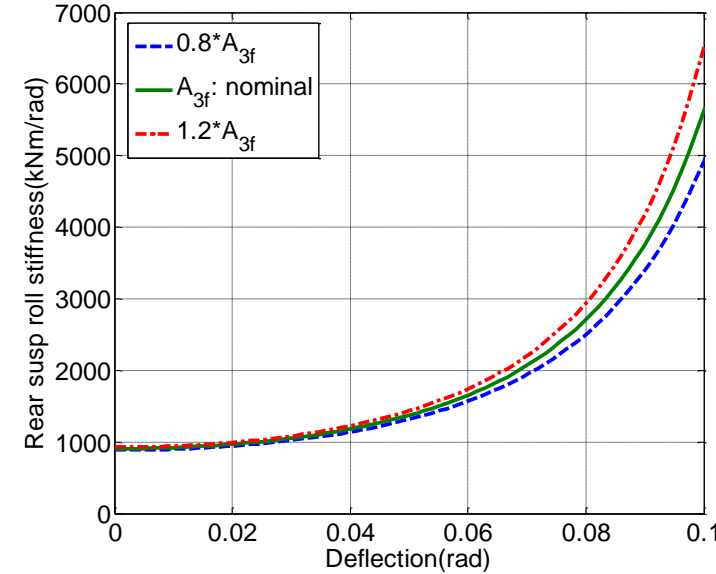

(b)

Fig. 21: Influence of variations in the front-strut annular area $A_{3 f}$ on the roll-mode stiffness of the hydraulically-coupled suspension C1: (a) front suspension roll stiffness; and (b) rear suspension roll stiffness.

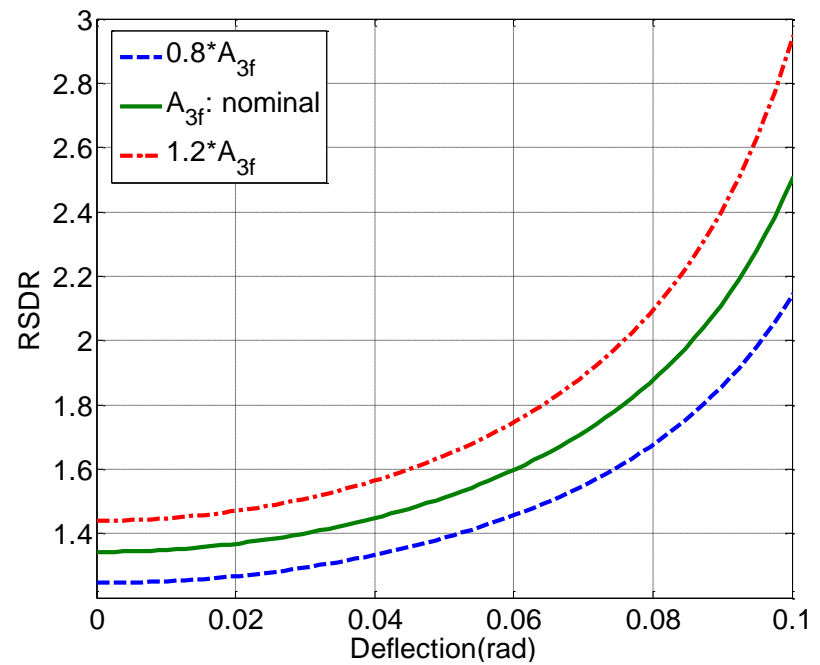

Fig. 22: Influence of variations in $A_{3 f}$ on the roll stiffness distribution ratio (RSDR) of the hydraulically-coupled suspension $\mathrm{C} 1$.

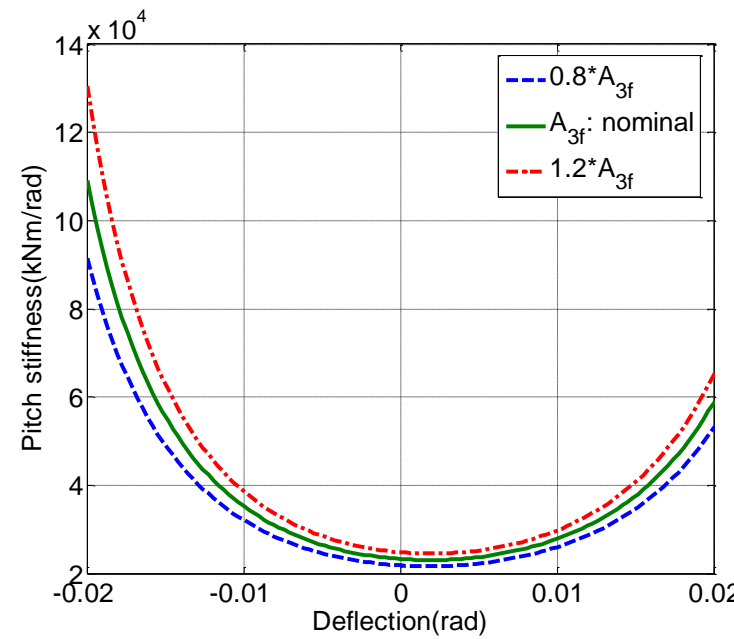

(a)

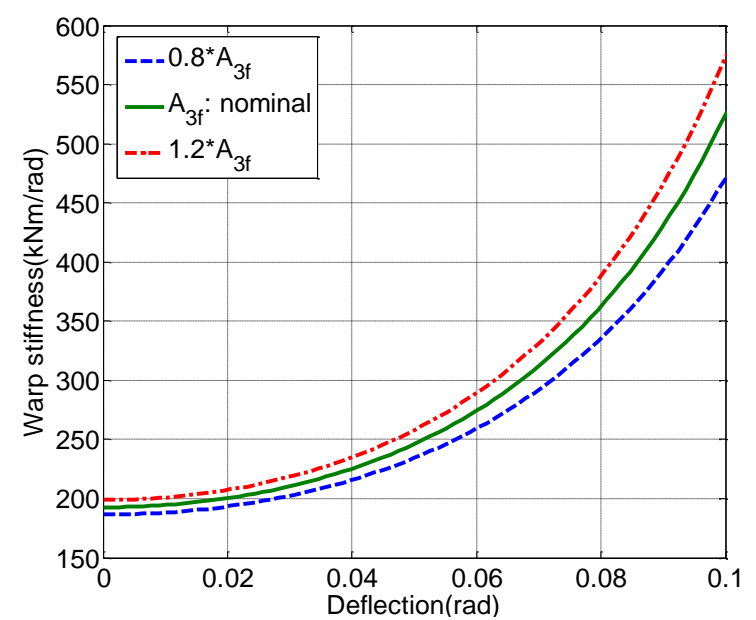

(b)

Fig. 23: Influence of variations in $A_{3 f}$ on: (a) pitch stiffness; and (b) warp stiffness of the hydraulically-coupled suspension $\mathrm{C} 1$. 